\title{
Ground state solutions to a class of critical Schrödinger problem
}

https://doi.org/10.1515/anona-2020-0192

Received December 3, 2020; accepted May 14, 2021.

Abstract: We consider the following critical nonlocal Schrödinger problem with general nonlinearities

$$
\left\{\begin{array}{l}
-\left(\varepsilon^{2} a+\varepsilon b \int_{\mathbb{R}^{3}}|\nabla u|^{2}\right) \Delta u+V(x) u=f(u)+u^{5}, \quad x \in \mathbb{R}^{3}, \\
u \in H^{1}\left(\mathbb{R}^{3}\right),
\end{array}\right.
$$

and study the existence of semiclassical ground state solutions of Nehari-Pohožaev type to $\left(S K_{\varepsilon}\right)$, where $f(u)$ may behave like $|u|^{q-2} u$ for $q \in(2,4]$ which is seldom studied. With some decay assumption on $V$, we establish an existence result which improves some exiting works which only handle $q \in(4,6)$. With some monotonicity condition on $V$, we also get a ground state solution $\bar{v}_{\varepsilon}$ and analysis its concentrating behaviour around global minimum $x_{\varepsilon}$ of $V$ as $\varepsilon \rightarrow 0$. Our results extend some related works.

Keywords: Kirchhoff-Schrödinger equation; Ground state; Pohožaev identity

MSC: 35J05; 35J20; 35J60

\section{Introduction and main results}

In this paper, we consider the following nonlocal Schrödinger equation

$$
\begin{cases}-\left(\varepsilon^{2} a+\varepsilon b \int_{\mathbb{R}^{3}}|\nabla u|^{2}\right) \Delta u+V(x) u=f(u)+u^{5}, & x \in \mathbb{R}^{3}, \\ u \in H^{1}\left(\mathbb{R}^{3}\right), & x \in \mathbb{R}^{3},\end{cases}
$$

where $\varepsilon, a, b>0$. Such nonlocal equation is also called Kirchhoff-Schrödinger equation. We make the following assumptions on potential function and nonlinear term

(V1) $V \in C\left(\mathbb{R}^{3}, \mathbb{R}\right)$ and $0<V_{0}=\min _{x \in \mathbb{R}^{3}} V(x)<V_{\infty}=\lim _{|y| \rightarrow \infty} V(y)$;

(F1) $f \in C(\mathbb{R}, \mathbb{R})$ and $\lim _{t \rightarrow 0} \frac{f(t)}{t}=0, \lim _{|t| \rightarrow \infty} \frac{f(t)}{t^{5}}=0$;

(F2) there exist constants $\mu, \mu_{1}>0$ and $q \in(2,4]$ such that $F(t) \geqslant \mu t^{q}-\mu_{1} t^{2}$ for all $t \geq 0$, where $F(t)=$ $\int_{0}^{t} f(s) \mathrm{d} s$;

(F3) there exists a constant $p \in(2,6)$ such that $\frac{f(t) t+6 F(t)}{|t|^{p-1} t}$ is nondecreasing on $(-\infty, 0) \cup(0,+\infty)$.

In [39], Zhang, Chen and Zou used (V1) (F2) to study the existence of standing waves to nonlinear Schrödinger equations involving critical growth, and (V1) (F2) was also used by S. Chen et al. in [6] to study the ground state solutions to Schrödinger-Poisson system. (F3) once appeared in [36] and is weaker than the

*Corresponding Author: Anmin Mao, School of Mathematical Sciences, Qufu Normal University, Shandong 273165, P.R. China, E-mail: maoam@163.co

Shuai Mo, School of Mathematical Sciences, Qufu Normal University, Shandong 273165, P.R. China, E-mail: 1063123691@qq.com 
following weak version of Nehari type condition

$$
f(t) / t \text { is nondecreasing on }(-\infty, 0) \cup(0,+\infty) .
$$

Problem $\left(S K_{\varepsilon}\right)$ is related to the stationary analogue of the following equation

$$
u_{t t}-\left(a+b \int_{\mathbb{R}^{3}}|\nabla u|^{2} \mathrm{~d} x\right) \Delta u=g(x, t),
$$

which is proposed by Kirchhoff [20] as an extension of classical D'Alembert's wave equation. It has been applied widely to model various physics problems and appears in some biological systems. For more details and backgrounds, we refer the reader to [2-4] and references therein. Owing to the presence of the term $\left(\int_{\mathbb{R}^{3}}|\nabla u|^{2} \mathrm{~d} x\right) \Delta u$, problem $\left(S K_{\varepsilon}\right)$ is no longer a pointwise identity, which makes the study of this question very complicated. However, it is worth mentioning that the pioneering work of Lions [24] introduced an abstract framework, and since then Kirchhoff type problem has received more and more attention from the mathematical community by using variational methods.

Making the scaling $u(x)=v(\varepsilon x),\left(S K_{\varepsilon}\right)$ is transformed to

$$
\begin{cases}-\left(a+b \int_{\mathbb{R}^{3}}|\nabla u|^{2}\right) \Delta u+V(\varepsilon x) u=f(u)+u^{5}, & x \in \mathbb{R}^{3}, \\ u \in H^{1}\left(\mathbb{R}^{3}\right), & x \in \mathbb{R}^{3},\end{cases}
$$

$u$ resolves $\left(P K_{\varepsilon}\right)$ iff $v$ resolves $\left(S K_{\varepsilon}\right)$. It follows from (V1) and (F1) that $\left(P K_{\varepsilon}\right)$ has a variational structure, which means the weak solutions of $\left(P K_{\varepsilon}\right)$ are the critical points of the $\mathcal{C}^{1}$ functional $\mathcal{J}_{\varepsilon}: H^{1}\left(\mathbb{R}^{3}\right) \rightarrow \mathbb{R}$ defined by

$$
\mathcal{J}_{\mathcal{E}}(u)=\frac{1}{2} \int_{\mathbb{R}^{3}}\left[a|\nabla u|^{2}+V(\varepsilon x) u^{2}\right] \mathrm{d} x+\frac{b}{4}\left(\int_{\mathbb{R}^{3}}|\nabla u|^{2} \mathrm{~d} x\right)^{2}-\int_{\mathbb{R}^{3}}\left[F(u)+\frac{1}{6} u^{6}\right] \mathrm{d} x .
$$

For simplicity, inspired by [6, 39], we set

$$
0 \in \Omega_{0}:=\left\{x \in \mathbb{R}^{3}: V(x)=V_{0}=\min _{x \in \mathbb{R}^{3}} V(x)\right\} .
$$

Most recently, many authors are concerned with semiclassical problems like $\left(S K_{\varepsilon}\right)$, i.e. the parameter $\varepsilon$ goes to zero. For $\varepsilon>0$ small, the solutions are called semiclassical states, which possess an important physical interest in describing the translation from quantum to classical mechanics. There are some valuable results on semiclassical solutions for Kirchhoff-type problems like $\left(S K_{\varepsilon}\right)$, we refer to [13, 15-17, 37].

Set $\varepsilon=1, V(x) \equiv 0$, and replace $\mathbb{R}^{3}$ with a bounded domain $\Omega$, then problem $\left(S K_{\varepsilon}\right)$ is related to the following problem

$$
\begin{cases}-\left(a+b \int_{\mathbb{R}^{3}}|\nabla u|^{2}\right) \Delta u=f(x, u), & x \in \Omega, \\ u=0, & x \in \partial \Omega .\end{cases}
$$

By minimax methods and invariant sets of descent flow, Mao and Zhang [28], Perera and Zhang [30] proved the existence of sign-changing solutions. At the same time, [1] obtained the existence of positive solution using variational methods when $f$ is critical growth.

When $\varepsilon>0$, the existence and qualitative properties of solutions to

$$
\left\{\begin{array}{l}
-\left(\varepsilon^{2} a+\varepsilon b \int_{\mathbb{R}^{3}}|\nabla u|^{2}\right) \Delta u+V(\varepsilon x)=f(u), \quad x \in \mathbb{R}^{3}, \\
u \in H^{1}\left(\mathbb{R}^{3}\right),
\end{array}\right.
$$


have been extensively studied; see for example $[6,9,10,18,26,34,37]$. If $f(t)$ is super-linear at $t=0$ and super-cubic at $t=\infty$, He and Zou [17] firstly studied $\left(\widetilde{S K}_{\varepsilon}\right)$ via the Mountain Pass Theorem and the Nehari manifold approach, under the condition that $f \in \mathcal{C}^{1}\left(\mathbb{R}^{+}, \mathbb{R}^{+}\right)$satisfies the Ambrosetti-Rabinowitz condition ((AR) in short)

$$
\exists \mu>4, \quad 0<\mu F(t) \leq f(t) t, \quad t \neq 0
$$

and the monotonicity condition((MN) in short)

$$
f(t) / t^{3} \text { is strictly increasing for } t \in(-\infty, 0) \cup(0,+\infty) .
$$

If $f(t)$ is not super-cubic at $t=\infty$, following the idea of Ruiz [31], Li and Ye [22] proved the special case that $V=1$ and $f(u)=|u|^{s-2} u$ for $3<s<6$ has a positive solution by using a new manifold related to Nehari equation and Pohožaev equality. Then, Guo [14] and Tang and Chen [36] improve the above results with more general $V$ and $f$, which handles the case where $f(u)$ behaves like $|u|^{s-2} u$ for $2<s \leq 3$.

For the critical case, Wang, Tian, Xu and Zhang [37] considered the problem

$$
-\left(\varepsilon^{2} a+\varepsilon b \int_{\mathbb{R}^{3}}|\nabla u|^{2}\right) \Delta u+V(x) u=\lambda g(u)+|u|^{4} u \text { in } \mathbb{R}^{3},
$$

under the assumption that $g \in \mathcal{C}^{1}\left(\mathbb{R}^{+}, \mathbb{R}^{+}\right)$is subcritical growth, $g(t)=o\left(t^{3}\right)$ as $t \rightarrow 0$ and $g(t) / t^{3}$ is strictly increasing on $(0, \infty)$. Inspired by [17], they obtained the existence, multiplicity and concentration of solutions when $\varepsilon>0$ small enough and $\lambda>0$ is large enough, in addition, they extended the results of [17] to the critical case. In [18], He and Zou also obtained the similar results relaying on (AR) and (MN). Based on the work of $[11,17]$, Liu and Guo [22] obtained the existence and concentration of positive ground state solution for $\left(S K_{\varepsilon}\right)$, where $f(u)+u^{5}$ is replaced by $\lambda K(x)|u|^{s-2} u+Q(x)|u|^{4} u$ for $4 \leq s<6$ with $K, Q \in \mathcal{C}\left(\mathbb{R}^{3}, \mathbb{R}\right)$ satisfying some suitable conditions.

We would like to emphasize that the previous work depends heavily on the condition (MN) or (AR) and can be applied to the case where $f(u) \sim|u|^{q-2} u$ for $4 \leq q<6$. Obviously, the approaches adopted in them do not work when $f$ satisfies neither (MN) nor (AR). Therefore, there are very few results concerning semiclassical ground state solutions for $\left(S K_{\varepsilon}\right)$ where $f(u)$ behaves like $|u|^{q-2} u$ for $q \in(2,4]$. The first purpose of this paper is to consider this case and improve some previous results.

To state our results, we need introduce the following decay assumption on $V$

(V2) $V \in C^{1}\left(\mathbb{R}^{3}, \mathbb{R}\right)$, and $2 \nabla V(x) \cdot x \leqslant(p-2) V(x)$ for all $x \in \mathbb{R}^{3}$, where $p$ is given by (F3).

Theorem 1.1. Assume that $V$ and $f$ satisfy (V1), (V2) and (F1) - (F3). Then there exist positive constants $\varepsilon \star$ and $\mu_{0}$ such that for $\varepsilon \in\left(0, \varepsilon_{\star}\right]$ and $\mu \geq \mu_{0}$, problem $\left(S K_{\varepsilon}\right)$ admits a ground state solution.

Next we consider the concentration of ground solution of $\left(S K_{\varepsilon}\right)$ as $\varepsilon \rightarrow 0$. Furthermore, we establish the exponential decay property of the solution obtained in the following theorem. For this reason, inspired by [36], we introduce the following monotonicity condition on $\mathrm{V}$

(V3) $V \in C^{1}\left(\mathbb{R}^{3}, \mathbb{R}\right)$, and $t \rightarrow \frac{4 V(t x)+\nabla V(t x) \cdot(t x)}{t^{(p-2) / 2}}$ is nonincreasing on $(0,+\infty)$ for any $x \in \mathbb{R}^{3} \backslash\{0\}$

which is different from the following monotonicity condition used in [6]:

$$
V \in C^{1}\left(\mathbb{R}^{3}, \mathbb{R}\right), t \mapsto t^{2}[V(t x)-\nabla V(t x) \cdot(t x)] \text { is nonincreasing on }(0,+\infty), x \in \mathbb{R}^{3} \backslash\{0\} .
$$

For $\varepsilon \geq 0$, we define the Pohožaev type functional $\mathcal{P}_{\varepsilon}$ as follows

$$
\mathcal{P}_{\varepsilon}(u)=\frac{a}{2}\|\nabla u\|_{2}^{2}+\frac{1}{2} \int_{\mathbb{R}^{3}}[3 V(\varepsilon x)+\nabla V(\varepsilon x) \cdot(\varepsilon x)] u^{2} \mathrm{~d} x+\frac{b}{2}\|\nabla u\|_{2}^{4}-3 \int_{\mathbb{R}^{3}}\left[F(u)+\frac{1}{6} u^{6}\right] \mathrm{d} x .
$$

Based on the fact that any solution $u$ of $\left(P K_{\varepsilon}\right)$ satisfies $\mathcal{P}_{\varepsilon}(u)=0$ and motivated by [22], define the following Nehari-Pohožaev functional on $H^{1}\left(\mathbb{R}^{3}\right)$ 


$$
\begin{aligned}
\mathcal{J}_{\varepsilon}(u)= & \frac{1}{2}\left\langle\mathcal{J}_{\varepsilon}^{\prime}(u), u\right\rangle+\mathcal{P}_{\varepsilon}(u) \\
= & a\|\nabla u\|_{2}^{2}+\frac{1}{2} \int_{\mathbb{R}^{3}}[4 V(\varepsilon x)+\nabla V(\varepsilon x) \cdot(\varepsilon x)] u^{2} \mathrm{~d} x \\
& +b\|\nabla u\|_{2}^{4}-\frac{1}{2} \int_{\mathbb{R}^{3}}[f(u) u+6 F(u)] \mathrm{d} x-\int_{\mathbb{R}^{3}} u^{6} \mathrm{~d} x,
\end{aligned}
$$

and set

$$
\mathcal{M}_{\varepsilon}:=\left\{u \in H^{1}\left(\mathbb{R}^{3}\right) \backslash\{0\}: \partial_{\varepsilon}(u)=0\right\},
$$

i.e., the Nehari-Pohožaev manifold of $\mathcal{J}_{\varepsilon}$. So every non-trivial solution of $\left(P K_{\varepsilon}\right)$ is contained in $\mathcal{M}_{\varepsilon}$. A nontrivial solution $\bar{u}$ of $\left(P K_{\varepsilon}\right)$ is called a ground state solution of Nehari-Pohožaev type if $\bar{u}$ satisfies $\mathcal{J}_{\varepsilon}(\bar{u})=$ $\inf _{u \in \mathcal{M}_{\varepsilon}} \mathcal{J}_{\varepsilon}(u)$.

Our second result is given as follows.

Theorem 1.2. Assume (V1), (V3) and (F1) - (F3) hold. Then there exists $\varepsilon_{0}>0$ determined by $V$ and $f$ such that for $\varepsilon \in\left(0, \varepsilon_{0}\right]$ and for $\mu \geq \mu_{0}$, where $\mu_{0}$ is given in Theorem 1.1, problem $\left(S K_{\varepsilon}\right)$ has a ground state solution $\bar{v}_{\varepsilon} \in H^{1}\left(\mathbb{R}^{3}\right) \backslash\{0\}$ such that $\bar{u}_{\varepsilon}(x)=\bar{v}_{\varepsilon}(\varepsilon x)$ satisfies

$$
\mathcal{J}_{\varepsilon}\left(\bar{u}_{\varepsilon}\right)=\inf _{u \in \mathcal{M}_{\varepsilon}} \mathcal{J}_{\varepsilon}(u)=\inf _{u \in H^{1}\left(\mathbb{R}^{3}\right) \backslash\{0\}} \max _{t>0} \mathcal{J}_{\varepsilon}\left(t^{1 / 2} u(\cdot / t)\right) .
$$

Moreover, the following statements hold

(i) for $\varepsilon \in\left(0, \varepsilon_{0}\right]$, there is a maximum point $x_{\varepsilon}$ of $\left|\bar{v}_{\varepsilon}\right|$ which satisfies that

$$
\lim _{\varepsilon \rightarrow 0} V\left(x_{\varepsilon}\right)=V_{0}=\min _{x \in \mathbb{R}^{3}} V(x) ;
$$

(ii) there exist $\Pi_{0}, \kappa_{0}$ independent of $\varepsilon \in\left(0, \varepsilon_{0}\right]$ such that the maximum point $x_{\varepsilon}$ of $\left|\bar{v}_{\varepsilon}\right|$ satisfies that

$$
\left|\bar{v}_{\varepsilon}(x)\right| \leq \Pi_{0} \exp \left(-\frac{\kappa_{0}}{\varepsilon}\left|x-x_{\varepsilon}\right|\right), \forall x \in \mathbb{R}^{3}, \quad \varepsilon \in\left(0, \varepsilon_{0}\right]
$$

(iii) for any sequence $\varepsilon_{n} \rightarrow 0, \bar{v}_{\varepsilon_{n}}\left(\varepsilon_{n} x+\chi_{\varepsilon_{n}}\right)$ converges in $H^{1}\left(\mathbb{R}^{3}\right)$ to a ground state solution of the following problem

$$
\begin{cases}-\left(a+b \int_{\mathbb{R}^{3}}|\nabla u|^{2}\right) \Delta u+V_{0} u=f(u)+u^{5}, & x \in \mathbb{R}^{3}, \\ u \in H^{1}\left(\mathbb{R}^{3}\right) . & x \in \mathbb{R}^{3} .\end{cases}
$$

It's worthy noting that (F3) (V3) are different from that in [6], and unlike [36], we just assume in (V1) that $\inf _{x \in \mathbb{R}^{3}} V(x)<V_{\infty}$ instead of $V(x) \leq V_{\infty}$ for all $x \in \mathbb{R}^{3}$. Owing to the critical term, we have to face the lack of compactness. To resolve the obstacle caused by the lack of compactness, we compare the mountain pass level with the minimax level of the associated limiting problem. For this purpose, we study the existence of ground solutions to the following equation

$$
\begin{cases}-\left(a+b \int_{\mathbb{R}^{3}}|\nabla u|^{2}\right) \Delta u+V u=f(u)+u^{5}, & x \in \mathbb{R}^{3}, \\ u \in H^{1}\left(\mathbb{R}^{3}\right), \quad u>0, & x \in \mathbb{R}^{3},\end{cases}
$$

where $V$ is a parameter with $0<V \leq V_{\max }:=\sup _{x \in \mathbb{R}^{3}} V(x)$. We have the following statement.

Theorem 1.3. Assume that $f$ satisfies (F1)-(F3), then $\left(K_{V}\right)$ possesses a ground state solution $\hat{u}_{V} \in H^{1}\left(\mathbb{R}^{3}\right) \backslash\{0\}$ for $\mu \geq \mu_{0}$, satisfying the following property

$$
I_{V}\left(\hat{u}_{V}\right)=\hat{m}_{V}:=\inf _{u \in \hat{\mathcal{M}}_{V}} I_{V}(u)=\inf _{u \in H^{1}\left(\mathbb{R}^{3}\right) \backslash\{0\}} \max _{t>0} I_{V}\left(t^{1 / 2}(u)_{t}\right)
$$


where $(u)_{t}(x):=u(x / t)$ for all $x \in \mathbb{R}^{3}$ and $t>0$, and

$$
\begin{gathered}
I_{V}(u):=\frac{1}{2} \int_{\mathbb{R}^{3}}\left(a|\nabla u|^{2}+V u^{2}\right) \mathrm{d} x+\frac{b}{4}\left(\int_{\mathbb{R}^{3}}|\nabla u|^{2} \mathrm{~d} x\right)^{2}-\int_{\mathbb{R}^{3}}\left[F(u)+\frac{1}{6} u^{6}\right] \mathrm{d} x \\
J_{V}(u)=a\|\nabla u\|_{2}^{2}+2 V\|u\|_{2}^{2}+b\|\nabla u\|_{2}^{4}-\frac{1}{2} \int_{\mathbb{R}^{3}}[f(u) u+6 F(u)] \mathrm{d} x-\int_{\mathbb{R}^{3}} u^{6} \mathrm{~d} x \\
\hat{\mathcal{M}}_{V}:=\left\{u \in H^{1}\left(\mathbb{R}^{3}\right) \backslash\{0\}: J_{V}(u)=0\right\} .
\end{gathered}
$$

The main difficulties lies in two aspects: (i) The fact that $f(u)$ does not satisfy (AR) condition nor (MN) condition prevents us from obtaining a bounded (PS) sequence and from using the Nehari manifold. (ii) The unboundedness of $\mathbb{R}^{3}$ and the presence of critical term $u^{5}$ result in the lack of compactness.

Motivated by [6], we firstly consider $\left(K_{V}\right)$ and prove Theorem 1.3 to find semiclassical solutions of $\left(S K_{\varepsilon}\right)$. Based on the general minimax principle [21, Proposition 2.8], we construct a Cerami sequence $\left\{u_{n}\right\}$ with $I_{V}\left(u_{n}\right) \rightarrow c_{V}$ and with the extra property that $J_{V}\left(u_{n}\right) \rightarrow 0$ which is crucial to deduce the boundedness of $\left\{u_{n}\right\}$, even the (AR) condition is not satisfied. By using some new estimates and subtle analysis introduced in [6], we show that $c_{V}<\frac{a b S^{3}}{4}+\frac{b^{3} S^{6}}{24}+\frac{\left(b^{2} S^{4}+4 a S\right)^{3 / 2}}{24}$ (see Lemma 3.3). More specifically, we determine the lower bound $\mu_{0}$ in Theorem 1.3 (see Lemma 3.3) unlike [15].

To prove the existence of the semiclassical solutions, following the idea of Jeanjean [19](the so-called monotonicity trick) and using a new global compactness lemma of critical type developed by [22, 36], we construct a sequence $\left\{u_{n}\right\}$ of the exact critical points of nearby functionals $\mathcal{J}_{\mathcal{E}, \lambda_{n}}$ which satisfies $\lambda_{n} \rightarrow 1$ and $\mathcal{J}_{\varepsilon, \lambda_{n}}^{\prime}\left(u_{n}\right)=0$, where

$$
\mathcal{J}_{\mathcal{E}, \lambda}(u)=\mathcal{J}_{\mathcal{E}}(u)+(1-\lambda) \int_{\mathbb{R}^{3}}\left[F(u)+\frac{1}{6} u^{6}\right] \mathrm{d} x \text { for all } u \in H^{1}\left(\mathbb{R}^{3}\right) \text { and } \lambda \in[1 / 2,1] .
$$

and show that $\mathcal{J}_{\varepsilon, \lambda}$ satisfies the Palais-Smale condition because of $c_{\varepsilon, \lambda}<m_{\lambda}^{\infty}$, as proved in Lemma 4.4. The fact that $u_{n}$ is the exact critical point provides additional information related to Pohožaev identity, which is important to ensure the boundedness of $\left\{u_{n}\right\}$.

Remark 1.4 As mentioned above, unlike [36], we just assume $\inf _{x \in \mathbb{R}^{3}} V(x)<V_{\infty}$ instead of $V(x) \leq V_{\infty}$ for all $x \in \mathbb{R}^{3}$, which makes it difficult to show $c_{\varepsilon}<m_{\varepsilon}^{\infty}$. To overcome this obstacle, we use some new energy inequalities and some subtle analysis and find two constants $\varepsilon_{\star}>0$ and $\bar{\lambda} \in[1 / 2,1)$ determined by $V$ and $f$ (see Lemma 4.4) such that

$$
c_{\varepsilon, \lambda}<m_{\lambda}^{\infty}, \quad \forall \lambda \in(\bar{\lambda}, 1], \varepsilon \in\left[0, \varepsilon^{*}\right] .
$$

Remark 1.5 To obtain the concentration phenomenon for ground state solution to $\left(S K_{\varepsilon}\right)$ as $\varepsilon \rightarrow 0$, we introduce some new proof techniques due to [6] to overcome the obstacles caused by the lack of (AR) and (MN), which is different from the previous work. Our work extended the results of [23] to critical case.

In Sect. 2, we give some preliminaries and necessary lemmas. Sect. 3 is devoted to show the existence of the ground state solution for the limited problem $\left(K_{V}\right)$, and give the proof of Theorem 1.3. The proof of theorem 1.1 is given in Sect. 4. We investigate the existence and concentration of the ground state solution of Nehari-Pohožaev type and complete the Proof of Theorem 1.2 in Sect. 5 and Sect. 6.

\section{Preliminaries}

In this section, we give some preliminaries. We will make use of the following notations.

- $H^{1}\left(\mathbb{R}^{3}\right)$ denotes the usual Sobolev space equipped with the inner product and norm $(u, v)=\int_{\mathbb{R}^{3}}(\nabla u$. $\nabla v) \mathrm{d} x,\|u\|=(u, u)^{1 / 2}$ for all $u, v \in H^{1}\left(\mathbb{R}^{3}\right)$.

- $|u|_{q}:=\left(\int_{\mathbb{R}^{3}}|u|^{q}\right)^{\frac{1}{q}}$ for $1 \leq q<\infty$ and $u \in L^{q}\left(\mathbb{R}^{3}\right)$. 
- $C, C_{i}$ denote (possibly different) various positive constant.

- $S=\inf _{u \in H^{1}\left(\mathbb{R}^{3}\right) \backslash\{0\}}\|\nabla u\|_{2}^{2} /\|u\|_{2}^{2}$.

- $B_{r}(x):=\left\{y \in \mathbb{R}^{3}:|y-x|<r\right\}$ for any $x \in \mathbb{R}^{3}$ and $r>0$.

- $(u)_{t}(x):=u(x / t)$ for all $x \in \mathbb{R}^{3}$ and $t>0$ along any $u \in H^{1}\left(\mathbb{R}^{3}\right) \backslash\{0\}$.

It is easy to check that (V1) and (V3) implies that

$$
\begin{aligned}
h_{1}(t, y) & :=V(y)-t^{4} V(t y)-\frac{2\left(1-t^{(6+p) / 2}\right)}{6+p}[4 V(y)+\nabla V(y) \cdot y] \\
& \geq 0, \quad \forall t \geq 0, y \in \mathbb{R}^{3} \backslash\{0\} .
\end{aligned}
$$

Lemma 2.1. Assume that (F1) and (F3) hold.Then

$$
\begin{aligned}
h_{2}(t, \tau) & :=t^{3} F\left(t^{1 / 2} \tau\right)-F(\tau)+\frac{1-t^{(6+p) / 2}}{6+p}[f(\tau) \tau+6 F(\tau)] \\
& \geq 0, \quad \forall t \geq 0, \tau \in \mathbb{R} .
\end{aligned}
$$

Proof. It is easy to see that for all $t \geq 0, h_{2}(t, \tau) \geq 0$. For $\tau \neq 0$, by (F3), we have

$$
\begin{aligned}
\frac{\mathrm{d}}{\mathrm{d} t} h_{2}(t, \tau)= & \frac{1}{2} t^{2+p / 2}|\tau|^{p}\left[\frac{f\left(t^{1 / 2} \tau\right) t^{1 / 2} \tau+6 F\left(t^{1 / 2} \tau\right)}{\left|t^{1 / 2} \tau\right|^{p}}-\frac{f(\tau) \tau+6 F(\tau)}{|\tau|^{p}}\right] \\
& \begin{cases}\geq 0, & t \geq 1, \\
\leq 0, & t \leq 1,\end{cases}
\end{aligned}
$$

which, together with the continuity of $h_{2}(\cdot, \tau)$, implies that $h_{2}(t, \tau) \geq h_{2}(1, \tau)=0$ for all $t \geq 0$ and $\tau \notin \mathbb{R} \backslash\{0\}$. This shows (2.2) holds.

Lemma 2.2. Assume that (V1), (V2), (F1) and (F3) hold. Then

$$
\begin{aligned}
\mathcal{J}_{\varepsilon}(u) \geq \mathcal{J}_{\mathcal{E}}\left(t^{1 / 2}(u)_{t}\right)+\frac{2\left(1-t^{(6+p) / 2}\right)}{6+p} \mathcal{J}_{\varepsilon}(u) \\
+\frac{b}{4(6+p)} \alpha(t)\|\nabla u\|_{2}^{4}, \quad \forall u \in H^{1}\left(\mathbb{R}^{3}\right), t>0,
\end{aligned}
$$

where

$$
\alpha(t)=8 t^{(6+p) / 2}-(6+p) t^{4}+p-2, \quad \forall t \geq 0 .
$$

Proof. Since $p>2$, one can easily have

$$
\alpha(t)>0, \forall t \in[0,1) \cup(1, \infty) .
$$

By (1.1), one has

$$
\begin{aligned}
\mathcal{J}_{\varepsilon}\left(t^{1 / 2}(u)_{t}\right)= & \frac{a t^{2}}{2}\|\nabla u\|_{2}^{2}+\frac{t^{4}}{2} \int_{\mathbb{R}^{3}} V(t \varepsilon x) u^{2} \mathrm{~d} x+\frac{b t^{4}}{4}\|\nabla u\|_{2}^{4} \\
& -t^{3} \int_{\mathbb{R}^{3}} F\left(t^{1 / 2} u\right) \mathrm{d} x-\frac{1}{6} t^{6}\|u\|_{6}^{6}, \quad \forall u \in H^{1}\left(\mathbb{R}^{3}\right), t>0 .
\end{aligned}
$$

It is easy to check that

$$
\begin{gathered}
\frac{1-t^{2}}{2}-\frac{2\left(1-t^{(6+p)}\right)}{6+p} \geq 0, \quad \forall t>0 . \\
\frac{1}{6} t^{6}-\frac{1}{6}+\frac{2\left(1-t^{(6+p) / 2}\right)}{6+p} \geq 0, \quad \forall t>0
\end{gathered}
$$


From (1.1), (1.4), (2.1), (2.2), (2.4), (2.5), (2.6) and (2.7), one has

$$
\begin{aligned}
& \mathcal{J}_{\varepsilon}(u)-\mathcal{J}_{\varepsilon}\left(t^{1 / 2}(u)_{t}\right) \\
& =\frac{a\left(1-t^{2}\right)}{2}\|\nabla u\|_{2}^{2}+\frac{1}{2} \int_{\mathbb{R}^{3}}\left[V(\varepsilon x)-t^{4} V(t \varepsilon x) u^{2}\right] \mathrm{d} x+\frac{b\left(1-t^{4}\right)}{4}\|\nabla u\|_{2}^{4} \\
& +\int_{\mathbb{R}^{3}}\left[t^{3} F\left(t^{1 / 2}(u)_{t}\right)-F(u)\right] \mathrm{d} x+\frac{1}{6}\left(t^{6}-1\right)\|u\|_{6}^{6} \\
& =\frac{2\left(1-t^{(6+p) / 2}\right)}{6+p}\left\{a\|\nabla u\|_{2}^{2}+\frac{1}{2} \int_{\mathbb{R}^{3}}\left[4 V(\varepsilon x)+\nabla V(\varepsilon \chi) \cdot(\varepsilon \chi) u^{2}\right] \mathrm{d} x+b\|\nabla u\|_{2}^{4}\right. \\
& \left.-\frac{1}{2} \int_{\mathbb{R}^{3}}[f(u) u+6 F(u)] \mathrm{d} x-\|u\|_{6}^{6}\right\}+\left[\frac{1-t^{2}}{2}-\frac{2\left(1-t^{(6+p)}\right)}{6+p}\right] a\|\nabla u\|_{2}^{2} \\
& +\frac{1}{2} \int_{\mathbb{R}^{3}} h_{1}(t, \varepsilon x) u^{2} \mathrm{~d} x+\left[\frac{1-t^{4}}{4}-\frac{2\left(1-t^{(6+p) / 2}\right)}{6+p}\right] b\|\nabla u\|_{2}^{4} \\
& +\int_{\mathbb{R}^{3}}\left\{t^{3} F\left(t^{1 / 2} u\right)-F(u)+\frac{1-t^{(6+p) / 2}}{6+p}[f(u) u+6 F(u)]\right\} \mathrm{d} x \\
& +\left[\frac{1}{6} t^{6}-\frac{1}{6}+\frac{2\left(1-t^{(6+p) / 2}\right)}{6+p}\right]\|u\|_{6}^{6} \\
& \geq \frac{2\left(1-t^{6+p) / 2}\right)}{6+p} \partial_{\varepsilon}(u)+\frac{b \alpha(t)}{4(6+p)}\|\nabla u\|_{2}^{4}, \quad \forall u \in H^{1}\left(\mathbb{R}^{3}\right), t>0 .
\end{aligned}
$$

This shows (2.3).

Let $t \rightarrow 0$, in (2.3), we have

$$
\mathcal{J}_{\varepsilon}(u) \geq \frac{2}{6+p} \mathcal{J}_{\varepsilon}(u)+\frac{b(p-2)}{4(6+p)}\|\nabla u\|_{2}^{4}, \quad \forall u \in H^{1}\left(\mathbb{R}^{3}\right) .
$$

Lemma 2.2 gives the following corollary immediately.

Corollary 2.1. Assume that (V1), (V3), (F1) and (F3) hold.Then for $u \in \mathcal{M}_{\varepsilon}$

$$
\mathcal{J}_{\varepsilon}(u)=\max _{t>0} \mathcal{J}_{\varepsilon}\left(t^{1 / 2}(u)_{t}\right)
$$

Lemma 2.3. Assume that (V1), (V3) and (F1)-(F3) hold.Then for any $u \in H^{1}\left(\mathbb{R}^{3}\right) \backslash\{0\}$, there exists a unique $t_{u}>0$ such that $t_{u}^{1 / 2}(u)_{t_{u}} \in \mathcal{M}_{\varepsilon}$.

Proof. Let $\varepsilon>0$ and $u \in H^{1}\left(\mathbb{R}^{3}\right) \backslash\{0\}$ be fixed. Define the function $\zeta_{\varepsilon}(t)=\mathcal{J}_{\varepsilon}\left(t^{1 / 2}(u)_{t}\right)$ on $(0, \infty)$. By $(1.4)$ and (2.5), we have

$$
\begin{aligned}
\zeta_{\varepsilon}^{\prime}(t)=0 \Longleftrightarrow & a t^{2}\|\nabla u\|_{2}^{2}+\frac{1}{2} t^{4} \int_{\mathbb{R}^{3}}[4 V(t \varepsilon x)+\nabla V(t \varepsilon x) \cdot(t \varepsilon x)] u^{2} \mathrm{~d} x \\
& +b t^{4}\|\nabla u\|_{x}^{4}-\frac{1}{2} t^{3} \int_{\mathbb{R}^{3}}\left[f\left(t^{1 / 2} u\right)\left(t^{1 / 2} u\right)+6 F\left(t^{1 / 2} u\right)\right] \mathrm{d} x \\
& -t^{6}\|u\|_{6}^{6}=0 \\
\Longleftrightarrow & \partial_{\varepsilon}\left(t^{1 / 2}(u)_{t}\right)=0 \Longleftrightarrow t^{1 / 2}(u)_{t} \in \mathcal{M}_{\varepsilon} .
\end{aligned}
$$

By (F1) and (F2), we have $\lim _{t \rightarrow 0} \zeta_{\varepsilon}(t), \zeta_{\varepsilon}(t)>0$ for $t>0$ small and $\zeta_{\varepsilon}(t)<0$ for $t$ large. Therefore $\max _{t \in(0, \infty)} \zeta_{\varepsilon}(t)$ is achieved at $t_{u}>0$ so that $\zeta_{\varepsilon}^{\prime}\left(t_{u}\right)=0$ and $t_{u}^{1 / 2}(u)_{t_{u}} \in \mathcal{M}_{\varepsilon}$. 
Next, we claim that $t_{u}$ is unique for any $u \in H^{1}\left(\mathbb{R}^{3}\right) \backslash\{0\}$. If not, we can assume that there exist positive constants $t_{u, 1} \neq t_{u, 2}$ such that $\partial_{\varepsilon}\left(t_{u, 1}^{1 / 2}(u)_{t_{u, 1}}\right)=\partial_{\varepsilon}\left(t_{u, 2}^{1 / 2}(u)_{t_{u, 2}}\right)=0$, i.e. $t_{u, 1}^{1 / 2}(u)_{t_{u, 1}}, t_{u, 2}^{1 / 2}(u)_{t_{u, 2}} \in \mathcal{M}_{\varepsilon}$. Then (2.3) and (2.4) imply

$$
\begin{aligned}
\mathcal{J}_{\varepsilon}\left(t_{u, 1}^{1 / 2}(u)_{t_{u, 1}}\right) & \geq \mathcal{J}_{\varepsilon}\left(t_{u, 2}^{1 / 2}(u)_{t_{u, 2}}\right)+\frac{2\left(t_{u, 1}^{(6+p) / 2}(u)_{t_{u, 1}}-t_{u, 2}^{(6+p) / 2}(u)_{t_{u, 2}}\right)}{(6+p) t_{u, 1}^{(6+p) / 2}} J_{\varepsilon}\left(t_{u, 1}^{1 / 2}(u)_{t_{u, 1}}\right) \\
& \geq \mathcal{J}_{\varepsilon}\left(t_{u, 1}^{1 / 2}(u)_{t_{u, 1}}\right)+\frac{2\left(t_{u, 2}^{(6+p) / 2}(u)_{t_{u, 2}}-t_{u, 1}^{(6+p) / 2}(u)_{t_{u, 1}}\right)}{(6+p) t_{u, 1}^{(6+p) / 2}} J_{\varepsilon}\left(t_{u, 2}^{1 / 2}(u)_{t_{u, 2}}\right) .
\end{aligned}
$$

This contradiction shows that $t_{u}>0$ is unique for any $u \in H^{1}\left(\mathbb{R}^{3}\right) \backslash\{0\}$.

Combining Corollary 2.1 with Lemma 2.3, we have the following lemma.

Lemma 2.4. Assume that (V1), (V3) and (F1)-(F3) hold. Then

$$
\inf _{u \in \mathcal{M}_{\varepsilon}} \mathcal{J}_{\mathcal{E}}(u)=m_{\varepsilon}=\inf _{u \in H^{1}\left(\mathbb{R}^{3}\right) \backslash\{0\}} \max _{t>0} \mathcal{J}_{\varepsilon}\left(t^{1 / 2}(u)_{t}\right) .
$$

Lemma 2.5. Assume that $V$ satisfies (V1) and (V3).Then there exists $\gamma_{1}>0$ independent of $\varepsilon>0$ such that

$$
2 a\|\nabla u\|_{2}^{2}+\int_{\mathbb{R}^{3}}[4 V(\varepsilon x)+\nabla V(\varepsilon x) \cdot(\varepsilon x)] u^{2} \mathrm{~d} x \geq \gamma_{1}\|u\|^{2}, \quad \forall u \in H^{1}\left(\mathbb{R}^{3}\right) .
$$

Proof. By (2.1), $\lim _{t \rightarrow 0} h_{1}(t, y) / t^{(6+p) / 2} \geq 0$ for any $y \in \mathbb{R}^{3} \backslash\{0\}$, which implies

$$
4 V(y)+\nabla V(y) \cdot y \geq 0, \quad \forall y \in \mathbb{R}^{3} \backslash\{0\} .
$$

Set $V_{\text {max }}:=\max _{x \in \mathbb{R}^{3}} V(x) \in(0, \infty)$, and $t_{0}=\left(2 V_{\max } / V_{0}\right)^{1 / 4}>1$. Then it follows from (V1), (2.1) and (2.12) that

$$
\begin{aligned}
\frac{2}{6+p}[4 V(y)+\nabla V(y) \cdot y] & \geq \frac{2\left(1-t_{0}^{-(6+p) / 2}\right)}{6+p}[4 V(y)+\nabla V(y) \cdot y] \\
& \geq t_{0}^{(2-p) / 2} V\left(t_{0} y\right)-t_{0}^{-(6+p) / 2} V(y) \\
& \geq t_{0}^{(2-p) / 2}\left[V_{0}-t_{0}^{-4} V_{\max }\right] \\
& =\frac{V_{0}}{2}\left(\frac{2 V_{\max }}{V_{0}}\right)^{(2-p) / 8}, \quad \forall y \in \mathbb{R}^{3} .
\end{aligned}
$$

Let $\gamma_{1}=\min \left\{2 a, \frac{V_{0}}{2}\left(\frac{2 V_{\max }}{V_{0}}\right)^{(2-p) / 8}\right\}$. Then (2.13) implies that (2.11) holds.

Lemma 2.6. Assume that (V1), (V3) and (F1)-(F3) hold. Then exist constants $\delta>0, \rho_{0}>0$ independent of $\varepsilon$ such that $\inf _{u \in \mathcal{M}_{\varepsilon}}\|\nabla u\|_{2}^{2}>\delta$ and $m_{\varepsilon}=\inf _{u \in \mathcal{M}_{\varepsilon}} \mathcal{J}_{\varepsilon}(u)>\rho_{0}$.

Proof. Since $\partial_{\varepsilon}(u)=0$ for all $u \in \mathcal{M}_{\varepsilon}$, by (F1), (1.4), (2.11) and Sobolev embedding inequality, there exists a constant $C_{0}>0$ independent of $\varepsilon$ such that

$$
\begin{aligned}
\gamma_{1}\|u\|^{2}+2 b\|\nabla u\|_{2}^{4} & \leq 2 a\|\nabla u\|_{2}^{2}+\int_{\mathbb{R}^{3}}[4 V(\varepsilon x)+\nabla V(\varepsilon x) \cdot(\varepsilon x)] \\
& =\int_{\mathbb{R}^{3}}[f(u) u+F(u)] \mathrm{d} x+2\|u\|_{6}^{6} \\
& \leq \frac{\gamma_{1}}{2}\|u\|_{2}^{2}+C_{0}\|u\|_{6}^{6} \\
& \leq \frac{\gamma_{1}}{2}\|u\|^{2}+C_{0} S^{-3}\|\nabla u\|_{2}^{6}
\end{aligned}
$$


which implies

$$
\|\nabla u\|_{2}^{2} \geq \delta:=\left(\frac{2 b S^{3}}{C_{0} \gamma_{1}}\right)^{1 / 2}, \quad \forall u \in \mathcal{M}_{\varepsilon} .
$$

By (2.9) and (2.14), one has

$$
\mathcal{J}_{\varepsilon}(u)=\mathcal{J}_{\varepsilon}(u)-\frac{2}{6+p} \mathcal{J}_{\varepsilon}(u) \geq \frac{b(p-2)}{4(6+p)}\|\nabla u\|_{2}^{2} \geq \frac{b(p-2)}{4(6+p)} \delta^{4}=\rho_{0}, \quad \forall u \in \mathcal{M}_{\varepsilon} .
$$

This completes the proof.

As the proof of [23, Lemma 2.9], we have the following statement.

Lemma 2.7. Assume that (V1), (V3) and (F1)-(F3) hold. If $\bar{u} \in \mathcal{M}_{\varepsilon}$ and $\mathcal{J}_{\varepsilon}(u)=m_{\varepsilon}$, then $\bar{u}$ is a critical point of $\mathcal{J}_{\varepsilon}$.

\section{Ground state solution for the limited problem}

In this section, by using the following general minimax principle, we show the existence of the ground state solution for $\left(K_{V}\right)$, and give the proof of Theorem 1.3. To prove the existence of nontrivial solutions, we use the following general minimax principle [21, Proposition 2.8], which is a stronger version of [38, Theorem 2.8].

Lemma 3.1. Let $X$ be a Banach space. Let $M_{0}$ be a closed subspace of the metric space $M$ and let $\Gamma_{0} \subset \mathcal{C}\left(M_{0}, X\right)$. Define

$$
\bar{\Gamma}:=\left\{\gamma \in \mathcal{C}\left(M_{0}, X\right):\left.\gamma\right|_{M_{0}} \in \Gamma_{0}\right\} .
$$

If $\varphi \in \mathcal{C}^{1}(X, \mathbb{R})$ verifies

$$
\infty>c:=\inf _{\gamma \in \Gamma} \sup _{u \in M} \varphi(\gamma(u))>b:=\sup _{\gamma_{0} \in \Gamma_{0}} \sup _{u \in M_{0}} \varphi\left(\gamma_{0}(u)\right),
$$

then, for every $\sigma \in(0,(c-b) / 2), \delta>0$ and $\gamma \in \bar{\Gamma}$ satisfying

$$
\sup _{M} \varphi \circ \gamma \leq c+\sigma,
$$

there exist $u \in X$ such that

(i) $c-2 \sigma \leq \varphi(u) \leq c+2 \sigma$,

(ii) $\operatorname{dist}(u, \gamma(M)) \leq 2 \delta$,

(iii) $(1+\|u\|)\left\|\varphi^{\prime}(u)\right\| \leq 8 \sigma / \delta$.

In the following, we apply Lemma 3.1 to obtain a Cerami sequence for the functional $I_{V}$ with $J_{V}\left(u_{n}\right) \rightarrow 0$.

Lemma 3.2. Assume that (F1) and (F2) hold. Then there exist a sequence $\left\{u_{n}\right\} \subset H^{1}\left(\mathbb{R}^{3}\right)$ satisfying

$$
I_{V}\left(u_{n}\right) \rightarrow c_{V}>0,\left\|I_{V}^{\prime}\left(u_{n}\right)\right\|(1+\|u\|) \rightarrow 0, \quad J_{V}\left(u_{n}\right) \rightarrow 0,
$$

where

$$
c_{V}:=\inf _{\gamma \in \Gamma} \max _{t \in[0,1]} I_{V}(\gamma(t)), \quad \Gamma:=\left\{\gamma \in \mathcal{C}\left([0,1], H^{1}\left(\mathbb{R}^{3}\right)\right): \gamma(0)=0, I_{V}(\gamma(1))<0\right\}
$$

Proof. By (F1), one has $I_{V}(t u) \rightarrow-\infty$, as $t \rightarrow \infty$ for every $u \in H^{1}\left(\mathbb{R}^{3}\right) \backslash\{0\}$. A standard argument shows that $\Gamma \neq \varnothing$ and $c_{V}<\infty$. Moreover, it is easy to see that there exist constants $\rho_{1}>0$ and $\sigma_{1}>0$ such that

$$
I_{V}(u) \geq 0 \text { for all } u \text {, with }\|u\| \leq \rho_{1} \text { and } I_{V}(u) \geq \sigma_{1} \text { for all } u \text {, with }\|u\|=\rho_{1} \text {. }
$$

Clearly $\gamma(0)=0$ and $I_{V}(\gamma(1))<0$ for every $\gamma \in \Gamma$. Hence (3.2) implies that $\|\gamma(1)\|>\rho_{1}$. There exists $t_{\gamma} \in(0,1)$ such that $\left\|\gamma\left(t_{\gamma}\right)\right\|=\rho_{1}$. Thus, we have

$$
\sup _{t \in[0,1]} I_{V}(\gamma(t)) \geq I_{V}\left(\gamma\left(t_{\gamma}\right)\right) \geq \sigma_{1}>0 \text { for all } \gamma \in \Gamma,
$$


which shows

$$
\infty>c_{V}=\inf _{\gamma \in \Gamma} \max _{t \in[0,1]} I_{V}(\gamma(t)) \geq \sigma_{1}>0 .
$$

Let us define the continuous map $h: \mathbb{R} \times H^{1}\left(\mathbb{R}^{3}\right) \rightarrow H^{1}\left(\mathbb{R}^{3}\right), h(s, v)(x)=e^{\frac{s}{2}} v\left(x / e^{s}\right)$ for $s \in \mathbb{R}, v \in H^{1}\left(\mathbb{R}^{3}\right)$ and $x \in \mathbb{R}^{3}$, where $\mathbb{R} \times H^{1}$ is a Banach space, with the product norm $\|(s, v)\|:=\left(|s|^{2}+\|v\|^{2}\right)^{(1 / 2)}$. We consider the following auxiliary functional

$$
\tilde{I}_{V}=I_{V}(h(s, u))=\frac{a e^{2 s}}{2}\|\nabla u\|_{2}^{2}+\frac{V}{2} e^{4 s}\|u\|_{2}^{2}+\frac{b}{4} e^{4 s}\|\nabla u\|_{2}^{4}-e^{3 s} \int_{\mathbb{R}^{3}} F\left(e^{\frac{s}{2}} u\right) \mathrm{d} x-\frac{1}{6} e^{6 s}\|u\|_{6}^{6} .
$$

It is easy to see that $\tilde{I}_{V} \in \mathcal{C}^{1}\left(\mathbb{R} \times H^{1}\left(\mathbb{R}^{3}\right), \mathbb{R}\right)$, and

$$
\partial_{s} \tilde{I}_{V}(s, u)=J_{V}(h(s, u)), \quad \partial_{u} \tilde{I}_{V}^{\prime}(s, u) w=I_{V}^{\prime}(h(s, u)) h(s, w),
$$

for all $s \in \mathbb{R}$ and $u, w \in H^{1}\left(\mathbb{R}^{3}\right)$.

Define the minimax value $\tilde{c}_{V}$ for $\tilde{I}_{V}$,

$$
\tilde{c}_{V}=\inf _{\tilde{\gamma} \in \tilde{\Gamma}} \max _{t \in[0,1]} \tilde{I}_{V}(\tilde{\gamma}(t)),
$$

where

$$
\tilde{\Gamma}=\left\{\tilde{\gamma} \in \mathcal{C}\left([0,1], \mathbb{R} \times H^{1}\left(\mathbb{R}^{3}\right)\right): \tilde{\gamma}(0)=(0,0), \tilde{I}_{V}(\tilde{\gamma}(1))<0\right\} .
$$

Since $\Gamma=\{h \circ \tilde{\gamma}: \tilde{\gamma} \in \tilde{\Gamma}\}$, it implies $c_{V}=\tilde{c}_{V}$. By the definition of $c_{V}$, for every $n \in \mathbb{N}$ there exists $\gamma_{n} \in \Gamma$ such that

$$
\max _{t \in[0,1]} \tilde{I}_{V}\left(0, \gamma_{n}(t)\right)=\max _{t \in[0,1]} I_{V}\left(\gamma_{n}(t)\right) \leq c_{V}+\frac{1}{n^{2}} .
$$

Then, we apply Lemma 3.1 to $\tilde{I}_{V}, M=[0,1], M_{0}=\{0,1\}$ and $X=\mathbb{R} \times H^{1}\left(\mathbb{R}^{3}\right)$. Let $\varepsilon_{n}=1 / n^{2}, \delta_{n}=1 / n$, and $\tilde{\gamma}_{n}(t)=\left(0, \gamma_{n}(t)\right)$. Since (3.3) implies $\varepsilon_{n}=1 / n^{2} \in\left(0, c_{V} / 2\right)$ for large $n \in \mathbb{N}$, Lemma 3.1 yields that existence of $\left(s_{n}, v_{n}\right) \in \mathbb{R} \times H^{1}\left(\mathbb{R}^{3}\right)$ such that, as $n \rightarrow \infty$,

$$
\begin{gathered}
\tilde{I}_{V}\left(s_{n}, v_{n}\right) \rightarrow c_{V}, \\
\left\|\tilde{I}_{V}\left(s_{n}, v_{v}\right)\right\|\left(1+\left\|\left(s_{n}, v_{n}\right)\right\|\right) \rightarrow 0, \\
\operatorname{dist}\left(\left(s_{n}, v_{n}\right),\{0\} \times \gamma_{n}([0,1])\right) \rightarrow 0 .
\end{gathered}
$$

Moreover, (3.5) gives that $s_{n} \rightarrow 0$. Note that for all $(\tau, w) \in \mathbb{R} \times H^{1}\left(\mathbb{R}^{3}\right)$,

$$
\left\langle\tilde{I}_{V}^{\prime}\left(s_{n}, v_{n},(\tau, w)\right)\right\rangle=\left\langle I_{V}^{\prime}\left(h\left(s_{n}, v_{n}\right)\right), h\left(s_{n}, v_{n}\right)\right\rangle+J_{V}\left(h\left(s_{n}, v_{n}\right)\right) \tau .
$$

Let $u_{n}:=h\left(s_{n}, v_{n}\right)$. Taking $\tau=1$ and $w=0$ in (3.6), we have $J_{V}\left(u_{n}\right) \rightarrow 0$ as $n \rightarrow \infty$. For every $v \in H^{1}\left(\mathbb{R}^{3}\right)$, set $\tau=0$ and $w_{n}=e^{-s_{n} / 2} v\left(x e^{s_{n}}\right)$ in (3.6), then (3.4) and (3.5) imply that

$$
\left|\left\langle\tilde{I}_{V}^{\prime}\left(u_{n}\right),\left(0, w_{n}\right)\right\rangle\right|\left(1+\left\|u_{n}\right\|\right)=\left|\left\langle I_{V}^{\prime}\left(h\left(s_{n}, v_{n}\right)\right), v\right\rangle\right|\left(1+\left\|\left(s_{n}, v_{n}\right)\right\|\right)=o(1)\left\|w_{n}\right\|
$$

as $n \rightarrow \infty$. This shows $\left\{u_{n}\right\}$ satisfies (3.1), as required.

Lemma 3.3. Assume that (F1) and (F2) hold. Then there exists $\mu_{0}>0$ such that for all $\mu \geq \mu_{0}$

$$
c_{V}<\frac{a b S^{3}}{4}+\frac{b^{3} S^{6}}{24}+\frac{\left(b^{2} S^{4}+4 a S\right)^{3 / 2}}{24} .
$$

Proof. For each $\varepsilon>0$, consider the function

$$
U_{\varepsilon}(x)=\frac{(3 \varepsilon)^{1 / 4}}{\left(\varepsilon+|x|^{2}\right)^{1 / 2}} \text { for all } x \in \mathbb{R}^{3} .
$$


Note that $U_{\varepsilon}$ is the extremal function for the embedding $\mathcal{D}^{1,2}\left(\mathbb{R}^{3}\right) \rightarrow L^{6}\left(\mathbb{R}^{3}\right)$ and

$$
S=\frac{\left\|U_{\varepsilon}\right\|_{2}^{2}}{\left\|U_{\varepsilon}\right\|_{6}^{2}}=\left(\frac{\sqrt{3}}{2}\right)^{2 / 3} \pi^{2} .
$$

Let $\eta \in \mathfrak{C}([0, \infty),[0,1])$ be such that

$$
\eta(r)= \begin{cases}r, & r \in[0,1), \\ 2-r, & r \in[1,2), \\ 0, & r \in[2, \infty) .\end{cases}
$$

Set $w_{\varepsilon}:=\eta(|x|) U_{\varepsilon}$ and $v_{\varepsilon}=w_{\varepsilon} /\left\|w_{\varepsilon}\right\|_{6}$. Inspired by [6], by a direct computation, for any $\varepsilon \in\left(0, \frac{\pi^{4 / 3}}{18}\right)$ we have

$$
\begin{gathered}
\left\|\nabla v_{\varepsilon}\right\|_{2}^{2} \leq S+C_{1} \varepsilon^{1 / 2}, \\
\left\|v_{\varepsilon}\right\|_{2}^{2} \leq C_{2} \varepsilon^{1 / 2}, \\
\left\|v_{\varepsilon}\right\|_{q}^{q} \geq C_{3} \varepsilon^{((6-q) / 4)},
\end{gathered}
$$

where $C_{1}=16(4 \ln 2-1) 6^{1 / 3}, C_{2}=16 \sqrt{3}, C_{3}=2^{(6-q) / 3} 3^{(q-6) / 6} \pi^{(2-q) / 2}$. From (F2), (1.7) and (3.9)-(3.11), one has, for all $t>0$

$$
\begin{aligned}
I_{V}\left(t v_{\varepsilon}\right) \leq & \frac{a t^{2}}{2}\left(S+C_{1} \varepsilon^{1 / 2}\right)+\frac{V_{\max }}{2} C_{2} \varepsilon^{1 / 2}+\frac{b t^{4}}{4}\left(S+C_{1} \varepsilon^{1 / 2}\right)^{2} \\
& -\mu t^{q} C_{3} \varepsilon^{(6-q) / 4}+\mu_{1} t^{2} C_{2} \varepsilon^{1 / 2}-\frac{1}{6} t^{6} \\
\leq & \frac{a S}{2} t^{2}+\frac{b S^{2}}{4} t^{4}-\frac{1}{6} t^{6}+\varepsilon^{1 / 2}\left[\left(a C_{1}+V_{\max } C_{2}+2 C_{2} \mu_{1}\right) \frac{t^{2}}{2}+\left(b C_{1}^{2}+2 b S C_{1}\right) \frac{t^{4}}{4}\right] \\
& -\mu t^{q} C_{3} \varepsilon^{(6-q) / 4} \\
= & \frac{t^{2}}{2}\left(a S+a C_{1} \varepsilon^{1 / 2}+V_{\max } C_{2}+2 \mu_{1} C_{2} \varepsilon^{1 / 2}\right)+\frac{t^{4}}{4}\left(b S^{2}+b C_{1}^{2} \varepsilon^{1 / 2}+2 b S C_{1} \varepsilon^{1 / 2}\right) \\
& -\frac{1}{6} t^{6}-\mu t^{q} C_{3} \varepsilon^{(6-q) / 4} .
\end{aligned}
$$

Then, define the functions on $[0, \infty)$

$$
\begin{gathered}
\eta_{1}(t)=\frac{a S}{2} t^{2}+\frac{b S^{2}}{4} t^{4}-\frac{1}{6} t^{6}, \\
\eta_{2}(t) \varepsilon^{1 / 2}=\left[\left(a C_{1}+V_{\max } C_{2}+2 C_{2} \mu_{1}\right) \frac{t^{2}}{2}+\left(b C_{1}^{2}+2 b S C_{1}\right) \frac{t^{4}}{4}\right] \varepsilon^{1 / 2}, \\
\eta_{3}(t)=-\mu t^{q} C_{3} \varepsilon^{(6-q) / 4} .
\end{gathered}
$$

It is easy to see that

$$
I_{V}\left(t v_{v}\right) \leq \eta_{1}(t)+\eta_{2}(t) \varepsilon^{1 / 2}+\eta_{3}(t) .
$$

Let $\varepsilon_{0}:=\min \left\{\frac{\pi^{4 / 3}}{18},\left(\frac{a S}{2 a C_{1}+2 V_{\max }+4 \mu_{1} C_{2}}\right)^{2},\left(\frac{b S^{2}}{2 b C_{1}^{2}}+4 b S C_{1}\right)^{2}\right\}$, for any $\varepsilon \in\left(0, \varepsilon_{0}\right]$, it is easily checked that $K(t)=\eta_{1}(t)+\eta_{2}(t) \varepsilon^{1 / 2}$ is increasing on $\left[0, t_{0} / \sqrt{2}\right]$ and decreasing on $\left[\sqrt{2} t_{0}, \infty\right]$, where $t_{0}=$ $\left(\frac{b S^{2}+\sqrt{b^{2} S^{4}+4 a S}}{2}\right)^{1 / 2}$.

We distinguish three cases.

1) $0<t \leq \frac{t_{0}}{\sqrt{2}}$. From (3.12), (3.13) and (3.15), we have for all $\varepsilon \in\left(0, \varepsilon_{0}\right]$

$$
I_{V}\left(t v_{\varepsilon}\right)<K(t) \leq K\left(\frac{t_{0}}{\sqrt{2}}\right)<\eta_{1}\left(t_{0}\right)-\frac{1}{2}\left[\eta_{1}\left(t_{0}\right)-\eta_{1}\left(\frac{t_{0}}{\sqrt{2}}\right)\right]+\eta_{2}\left(\frac{t_{0}}{\sqrt{2}}\right) \varepsilon^{1 / 2} .
$$


Let $\varepsilon_{1}:=\left(\frac{\eta_{1}\left(t_{0}\right)-\eta_{1}\left(\frac{t_{0}}{\sqrt{2}}\right)}{2 \eta_{2}\left(\frac{t_{0}}{\sqrt{2}}\right)}\right)^{2}$, and we have $I_{V}\left(t v_{\varepsilon}\right)<\eta_{1}\left(t_{0}\right)$ with $0<\varepsilon<\min \left\{\varepsilon_{0}, \varepsilon_{1}\right\}$ from (3.16). It shows

$$
\sup _{t \in\left(0, \frac{t_{0}}{\sqrt{2}}\right]} I_{V}\left(t v_{\varepsilon}\right)<\eta_{1}\left(t_{0}\right) \text { for all } \varepsilon \text {, with } 0<\varepsilon \leq \min \left\{\varepsilon_{0}, \varepsilon_{1}\right\} .
$$

2) $t \geq \sqrt{2} t_{0}$. In this case, (3.12), (3.13) and (3.15) imply that for all $\varepsilon \in\left(0, \varepsilon_{0}\right]$

$$
I_{V}\left(t v_{\varepsilon}\right) \leq K\left(\sqrt{2} t_{0}\right)<\eta_{1}\left(t_{0}\right)-\frac{1}{2}\left[\eta_{1}\left(t_{0}\right)-\eta_{1}\left(\sqrt{2} t_{0}\right)\right]+\eta_{2}\left(\sqrt{2} t_{0}\right) \varepsilon^{1 / 2}
$$

Let $\varepsilon_{2}:=\left(\frac{\eta_{1}\left(t_{0}\right)-\eta_{1}\left(\sqrt{2} t_{0}\right)}{2 \eta_{2}\left(\sqrt{2} t_{0}\right)}\right)^{2}$, and we have $I_{V}\left(t v_{\varepsilon}\right)<\eta_{1}\left(t_{0}\right)$ with $0<\varepsilon<\min \left\{\varepsilon_{0}, \varepsilon_{1}, \varepsilon_{2}\right\}$ from (3.18). It shows

$$
\sup _{t \in\left[\sqrt{2} t_{0}, \infty\right)} I_{V}\left(t v_{\varepsilon}\right)<\eta_{1}\left(t_{0}\right) \text { for all } \varepsilon \text {, with } 0<\varepsilon \leq \min \left\{\varepsilon_{0}, \varepsilon_{1}, \varepsilon_{2}\right\} .
$$

3) $\frac{t_{0}}{\sqrt{2}}<t<\sqrt{2} t_{0}$. Note that

$$
I_{V}\left(t v_{\varepsilon}\right)<\eta_{1}\left(t_{0}\right)+\eta_{2}\left(\sqrt{2} t_{0}\right) \varepsilon^{1 / 2}+\eta_{3}\left(\frac{t_{0}}{\sqrt{2}}\right)
$$

let $\varepsilon_{3}=\frac{2^{q / 2} \eta_{2}\left(\sqrt{2} t_{0}\right)}{\mu_{1} t_{0}^{q}}$ and $\varepsilon_{4}=\min \left\{\varepsilon_{1}, \varepsilon_{2}, \varepsilon_{3}\right\}$, then (3.20) implies that

$$
\sup _{t \in\left(\frac{t_{0}}{\sqrt{2}}, \sqrt{2} t_{0}\right)} I_{V}\left(t v_{\varepsilon_{4}}\right)<\eta\left(t_{0}\right),
$$

provided that $\mu \geq \mu_{0}$, with $\mu_{0}=\frac{\eta_{2}\left(\sqrt{2} t_{0}\right) 2^{q / 2}}{t_{0}^{q} C_{3}} \varepsilon_{4}^{(q-4) / 4}$.

It follows from (3.17), (3.19), (3.21), we have

$$
c_{V} \leq \sup _{t \geq 0} I_{V}\left(t v_{\varepsilon_{3}}\right)<\eta_{1}\left(t_{0}\right)=\frac{a b S^{3}}{4}+\frac{b^{3} S^{6}}{24}+\frac{\left(b^{2} S^{4}+4 a S\right)^{3 / 2}}{24}
$$

for all $\mu \geq \mu_{0}$. This completes the proof.

Lemma 3.4. Assume that (F1)-(F3) hold. Then $c_{V}=\hat{m}_{V}$.

Proof. From Lemma 2.4, we deduce that $c_{V} \leq \hat{m}_{V}$. Next, we show that $c_{V} \geq \hat{m}_{V}$. By (2.2),

$$
h_{2}(0, \tau)=\frac{1}{6+p}[f(\tau) \tau-p F(\tau)], \forall \tau \in \mathbb{R} .
$$

Note that for all $u \in H^{1}\left(\mathbb{R}^{3}\right)$,

$$
\begin{aligned}
I_{V}(u)-\frac{2}{6+p} J_{V}(u)= & \frac{(p+2) a}{2(6+p)}\|\nabla u\|_{2}^{2}+\frac{(p-2) b}{4(6+p)}\|\nabla u\|_{2}^{4}+\frac{p-2}{2(6+p)} V\|u\|_{2}^{2} \\
& +\frac{1}{6+p} \int_{\mathbb{R}^{3}}[f(u) u-p F(u)] \mathrm{d} x+\frac{6-p}{6(6+p)}\|u\|_{6}^{6} \\
\geq & \frac{(p+2) a}{2(6+p)}\|\nabla u\|_{2}^{2}+\frac{(p-2) b}{4(6+p)}\|\nabla u\|_{2}^{4}+\frac{p-2}{2(6+p)} V\|u\|_{2}^{2} \\
& +\frac{6-p}{6(6+p)}\|u\|_{6}^{6}
\end{aligned}
$$

by the definitions of $I_{V}, J_{V} \geq 0$ and (3.22). Since $\|\gamma(1)\|>\rho_{1}, I_{V}(\gamma(1))<0$ for all $\gamma \in \Gamma_{V}$, then (3.26) implies

$$
I_{V}(\gamma(1))-\frac{2}{6+p} J_{V}(\gamma(1))>0,
$$

which shows $J_{V}(\gamma(1))<0$. 
From $J_{V}(0)=0$, (F1) and $J_{V}(\gamma(1))<0$, there exist $\rho_{2} \in(0,\|\gamma(1)\|)$ and $\sigma_{2} \geq 0$ such that $J_{V}(u) \geq \sigma_{2}$ for all $\|u\|=\rho_{2}$, which implies that there exist $t_{V} \in(0,1)$ such that $J_{V}\left(\gamma\left(t_{V}\right)\right) \geq \sigma_{2}$. Thus, for every $\gamma \in \Gamma_{V}$ has to cross $\hat{\mathcal{M}}_{V}$, and $c_{V} \geq \hat{m}_{V}$. This shows that $c_{V}=\hat{m}_{V}$.

Proof of Theorem 1.3. In view of Lemma 3.2, there exist a sequence $\left\{u_{n}\right\} \subset H^{1}\left(\mathbb{R}^{3}\right)$ satisfying (3.1). By (3.1), (3.22) and (3.23), we have

$$
c_{V}+o(1)=I_{V}\left(u_{n}\right)-\frac{2}{6+p} J_{V}\left(u_{n}\right) \geq \frac{(p+2) a}{2(6+p)}\left\|\nabla u_{n}\right\|_{2}^{2}+\frac{p-2}{2(6+p)} V\left\|u_{n}\right\|_{2}^{2},
$$

which implies $\left\{u_{n}\right\}$ is bounded in $H^{1}\left(\mathbb{R}^{3}\right)$. Next, we claim that

$$
\limsup \sup _{n \rightarrow \infty} \int_{y \in \mathbb{R}^{3}}\left|u_{n}\right|^{2} \mathrm{~d} x>0 .
$$

If not, then Lions' concentration compactness principle implies that $u_{n} \rightarrow 0$ in $L^{s}\left(\mathbb{R}^{3}\right)$ for all $s \in(2,6)$. By (F1), we have for every $\varepsilon>0$, there exist constant $C_{\varepsilon}$ such that

$$
|f(\tau) \tau|+|F(\tau)| \leq \varepsilon\left(\tau^{2}+\tau^{6}\right)+C_{\varepsilon}|\tau|^{4} \text { for all } \tau \in \mathbb{R} .
$$

Thus, as $n \rightarrow \infty$

$$
o(1)=\left\langle I_{V}^{\prime}\left(u_{n}\right), u_{n}\right\rangle=a\left\|\nabla u_{n}\right\|_{2}^{2}+V\left\|u_{n}\right\|_{2}^{2}+b\left\|\nabla u_{n}\right\|_{2}^{4}-\left\|u_{n}\right\|_{6}^{6}+o(1)
$$

and

$$
c_{V}+o(1)=\frac{a}{2}\left\|\nabla u_{n}\right\|_{2}^{2}+\frac{V}{2}\left\|u_{n}\right\|_{2}^{2}+\frac{b}{4}\left\|\nabla u_{n}\right\|_{2}^{4}-\frac{1}{6}\left\|u_{n}\right\|_{6}^{6}+o(1)
$$

Since $\left\{u_{n}\right\}$ is bounded in $H^{1}\left(\mathbb{R}^{3}\right)$ and $c_{V}>0$, up to a subsequence, we may assume there exists constants $l_{1}, l_{2}>0$ such that

$$
a\left\|\nabla u_{n}\right\|_{2}^{2}+V\left\|u_{n}\right\|_{2}^{2} \rightarrow l_{1}, \quad b\left\|\nabla u_{n}\right\|_{2}^{4}, \quad n \rightarrow \infty .
$$

Together with (3.27), (3.28) and Sobolev inequality, we have

$$
\begin{gathered}
c_{V}=\frac{1}{3} l_{1}+\frac{1}{12} l_{2}, \\
a\left\|\nabla u_{n}\right\|_{2}^{2}+V\left\|u_{n}\right\| \geq a S\left\|u_{n}\right\|_{6}^{2}, \quad b\left\|\nabla u_{n}\right\|_{2}^{4} \geq b S^{2}\left\|u_{n}\right\|_{6}^{4} .
\end{gathered}
$$

Letting $n \rightarrow \infty$ in the above two inequalities, we achieve that

$$
\begin{gathered}
l_{1} \geq a S\left(l_{1}+l_{2}\right)^{1 / 3}, \\
l_{2} \geq\left(l_{1}+l_{2}\right)^{2 / 3} .
\end{gathered}
$$

Combining with (3.30) and (3.31), we have

$$
\left(l_{1}+l_{2}\right)^{1 / 3} \geq \frac{b S^{2}+\left(b^{2} S^{4}+4 a S\right)^{1 / 2}}{2}
$$

and

$$
\begin{aligned}
c_{V}=\frac{1}{3} l_{1}+\frac{1}{12} l_{2} & \geq \frac{1}{3}\left(l_{1}+l_{2}\right)^{1 / 3}+\frac{1}{12}\left(l_{1}+l_{2}\right)^{2 / 3} \\
& \geq \frac{a b S^{3}}{4}+\frac{b^{3} S^{6}}{24}+\frac{\left(b^{2} S^{4}+4 a S\right)^{3 / 2}}{24} .
\end{aligned}
$$

This contradiction shows that (3.25) holds. Thus there exist $\delta>0$ and a sequence $\left\{y_{n}\right\} \subset H^{1}\left(\mathbb{R}^{3}\right)$ such that $\int_{B_{1}\left(y_{n}\right)}\left|u_{n}\right|^{2} \mathrm{~d} x>\delta$. Let $\hat{u}_{n}=u_{n}\left(x+y_{n}\right)$. Then (3.1) gives

$$
I_{V}\left(\hat{u}_{n}\right) \rightarrow c_{V}>0, I_{V}^{\prime}\left(\hat{u}_{n}\right) \rightarrow 0, J_{V}\left(\hat{u}_{n}\right) \rightarrow 0 \text { as } n \rightarrow \infty
$$


and $\int_{B_{1}(0)}\left|\hat{u_{n}}\right|^{2} \mathrm{~d} x>\delta$ for all $n \in \mathbb{N}$. Therefore, there exists $\hat{u} \in H^{1}\left(\mathbb{R}^{3}\right) \backslash\{0\}$ such that, up to a subsequence,

$$
\left\{\begin{array}{l}
\hat{u}_{n} \rightarrow \hat{u} \text { in } H^{1}\left(\mathbb{R}^{3}\right), \\
\hat{u}_{n} \rightarrow \hat{u} \text { in } L_{l o c}^{s}\left(\mathbb{R}^{3}\right) \text { for all } s \in[1,6), \\
\hat{u}_{n} \rightarrow \hat{u} \text { a.e. on } \mathbb{R}^{3} .
\end{array}\right.
$$

Moreover, $\hat{u}$ is nontrivial, and $\hat{u}$ satisfies

$$
-\left(a+b A^{2}\right) \Delta \hat{u}+V \hat{u}=f(\hat{u})+\hat{u}^{5},
$$

where $A^{2}:=\lim _{n \rightarrow \infty}\left\|\nabla \hat{u}_{n}\right\|_{2}^{2}$ and $\|\nabla \hat{u}\|_{2}^{2} \leq A^{2}$. Hence, we have the following equalities

$$
\left\{\begin{array}{l}
\left(a+b A^{2}\right)\|\nabla \hat{u}\|_{2}^{2}+V\|\hat{u}\|_{2}^{2}=\int_{\mathbb{R}^{3}} f(\hat{u}) \hat{u} \mathrm{~d} x+\|\hat{u}\|_{6}^{6}, \\
\frac{1}{2}\left(a+b A^{2}\right)\|\nabla \hat{u}\|_{2}^{2}+\frac{3}{2} V\|\hat{u}\|_{2}^{2}=3 \int_{\mathbb{R}^{3}} F(\hat{u}) \mathrm{d} x+\frac{1}{2}\|\hat{u}\|_{6}^{6} .
\end{array}\right.
$$

Next, we show that $J_{V}(\hat{u})=0$. From (1.8) and (3.34), we have

$$
J_{V}(\hat{u})=b\|\nabla \hat{u}\|_{2}^{2}\left(\|\nabla \hat{u}\|_{2}^{2}-A^{2}\right) \leq 0 .
$$

If $J_{V}(\hat{u})<0$, it follows from Lemma 2.3, which is also true for $I_{V}$ and $J_{V}$, that there exist a unique $t \in(0,1)$ such that $J_{V}\left(t^{1 / 2}\left(\hat{u}_{t}\right)\right)=0$. Combining with (2.3), (3.22), (3.23), (3.32), the weak semicontinuity of norm, Fatou's lemma and Lemma 3.4 that

$$
\begin{aligned}
& \hat{m}_{V}= \lim _{n \rightarrow}\left[I_{V}\left(\hat{u}_{n}\right)-\frac{2}{6+p} J_{V}\left(\hat{u}_{n}\right)\right] \\
&= \frac{(p+2) a}{2(6+p)}\left\|\nabla \hat{u}_{n}\right\|_{2}^{2}+\frac{(p-2) b}{4(6+p)}\left\|\nabla \hat{u}_{n}\right\|_{2}^{4}+\frac{p-2}{2(6+p)} V\left\|\hat{u}_{n}\right\|_{2}^{2} \\
&+\frac{1}{6+p} \int_{\mathbb{R}^{3}}\left[f\left(\hat{u}_{n}\right) \hat{u}_{n}-p F\left(\hat{u}_{n}\right)\right] \mathrm{d} x+\frac{6-p}{6(6+p)}\left\|\hat{u}_{n}\right\|_{6}^{6} \\
& \geq \frac{(p+2) a}{2(6+p)}\|\nabla \hat{u}\|_{2}^{2}+\frac{(p-2) b}{4(6+p)}\|\nabla \hat{u}\|_{2}^{4}+\frac{p-2}{2(6+p)} V\|\hat{u}\|_{2}^{2} \\
&+\frac{1}{6+p} \int_{\mathbb{R}^{3}}[f(\hat{u}) \hat{u}-p F(\hat{u})] \mathrm{d} x+\frac{6-p}{6(6+p)}\|\hat{u}\|_{6}^{6} \\
&= I_{V}(\hat{u})-\frac{2}{6+p} J_{V}(\hat{u}) \\
& \geq I_{V}\left(t^{1 / 2}(\hat{u})_{t}\right)-\frac{2 t^{(6+p) / 2}}{6+p} J_{V}(u) \\
& \geq \hat{m}_{V}-\frac{2 t^{(6+p) / 2}}{6+p} J_{V}(u)>\hat{m}_{V},
\end{aligned}
$$

which is a contradiction. Hence we have $J_{V}(\hat{u})=0$ and $I_{V}(\hat{u})=\hat{m}_{V}$ which, together with Lemma 2.4 and Lemma 2.9, implies

$$
\begin{gathered}
I_{V}(\hat{u})=\hat{m}_{V}:=\inf _{u \in \hat{\mathcal{M}}_{V}} I_{V}(u)=\inf _{u \in H^{1}\left(\mathbb{R}^{3}\right) \backslash\{0\}} \max _{t>0} I_{V}\left(t^{1 / 2}(u)_{t}\right), \\
I_{V}^{\prime}(\hat{u})=0 .
\end{gathered}
$$

This completes the proof. 


\section{Existence of the ground state solutions}

In this section, by using the Jeanjean's monotonicity trick [19, Theorem 1.1], we show the existence of ground state solutions for $\left(S K_{\varepsilon}\right)$. To this end, for $\lambda \in[1 / 2,1]$, we introduce two families of functionals on $H^{1}\left(\mathbb{R}^{3}\right)$ defined by

$$
\mathcal{J}_{\varepsilon, \lambda}(u)=\frac{1}{2} \int_{\mathbb{R}^{3}}\left[a|\nabla u|^{2}+V(\varepsilon x) u^{2}\right] \mathrm{d} x+\frac{b}{4}\left(\int_{\mathbb{R}^{3}}|\nabla u|^{2} \mathrm{~d} x\right)^{2}-\lambda \int_{\mathbb{R}^{3}}\left[F(u)+\frac{1}{6} u^{6}\right] \mathrm{d} x,
$$

and

$$
\mathcal{J}_{\lambda}^{\infty}(u)=\frac{1}{2} \int_{\mathbb{R}^{3}}\left[a|\nabla u|^{2}+V_{\infty} u^{2}\right] \mathrm{d} x+\frac{b}{4}\left(\int_{\mathbb{R}^{3}}|\nabla u|^{2} \mathrm{~d} x\right)^{2}-\lambda \int_{\mathbb{R}^{3}}\left[F(u)+\frac{1}{6} u^{6}\right] \mathrm{d} x .
$$

In the same way as in [14], we can obtain the following lemma.

Lemma 4.1. Assume that (V1), (V2), (F1) and (F2) hold. Let $u$ be the critical points of $\mathcal{J}_{\varepsilon, \lambda}$ in $H^{1}\left(\mathbb{R}^{3}\right)$, then we have the following Pohožaev type identity

$$
\mathcal{P}_{\varepsilon, \lambda}(u)=\frac{a}{2}\|\nabla u\|_{2}^{2}+\frac{1}{2} \int_{\mathbb{R}^{3}}[3 V(\varepsilon x)+\nabla V(\varepsilon x) \cdot(\varepsilon x)] u^{2} \mathrm{~d} x+\frac{b}{2}\|\nabla u\|_{2}^{4}-3 \lambda \int_{\mathbb{R}^{3}}\left[F(u)+\frac{1}{6} u^{6}\right] \mathrm{d} x .
$$

Set $\mathcal{J}_{\varepsilon, \lambda}(u)=\frac{1}{2}\left\langle\mathcal{J}_{\varepsilon, \lambda}^{\prime}(u), u\right\rangle+\mathcal{P}_{\varepsilon, \lambda}(u)$ for all $\lambda \in[1 / 2,1]$, one has

$$
\begin{aligned}
\mathcal{J}_{\varepsilon, \lambda}(u)= & a\|\nabla u\|_{2}^{2}+\frac{1}{2} \int_{\mathbb{R}^{3}}[4 V(\varepsilon x)+\nabla V(\varepsilon x) \cdot(\varepsilon x)] u^{2} \mathrm{~d} x \\
& +b\|\nabla u\|_{2}^{4}-\frac{\lambda}{2} \int_{\mathbb{R}^{3}}[f(u) u+6 F(u)] \mathrm{d} x-\lambda \int_{\mathbb{R}^{3}} u^{6} \mathrm{~d} x .
\end{aligned}
$$

Correspondingly, for $\lambda \in[1 / 2,1]$ we also let

$$
\begin{gathered}
\mathcal{J}_{\lambda}^{\infty}(u)=a\|\nabla u\|_{2}^{2}+2 V_{\infty}\|u\|_{2}^{2}+b\|\nabla u\|_{2}^{2}-\frac{\lambda}{2} \int_{\mathbb{R}^{3}}[f(u) u+6 F(u)] \mathrm{d} x-\lambda\|u\|_{6}^{6}, \\
\mathcal{M}_{\lambda}^{\infty}=\left\{u \in H^{1}\left(\mathbb{R}^{3}\right) \backslash\{0\}: \partial_{\lambda}^{\infty}(u)=0\right\}, \quad m_{\lambda}^{\infty}=\inf _{u \in \mathcal{M}_{\lambda}^{\infty}} \mathcal{J}_{\lambda}^{\infty}(u) .
\end{gathered}
$$

Immediately, Lemma 2.2 implies the following lemma.

Lemma 4.2. Assume (F1), (F3) hold. Then

$$
\begin{aligned}
\mathcal{J}_{\lambda}^{\infty}(u) \geq \mathcal{J}_{\lambda}^{\infty}\left(t^{1 / 2}(u)_{t}\right)+\frac{2\left(1-t^{(6+p) / 2}\right)}{6+p} \mathcal{J}_{\lambda}^{\infty}(u) \\
\quad+\frac{b}{4(6+p)} \alpha(t)\left(2 V_{\infty}\|u\|_{2}^{2}+\|\nabla u\|_{2}^{4}\right), \quad \forall u \in H^{1}\left(\mathbb{R}^{3}\right), t>0 .
\end{aligned}
$$

For $\lambda \in[1 / 2,1]$, let

$$
\mathcal{J}_{\lambda}^{*}(u)=\frac{1}{2} \int_{\mathbb{R}^{3}}\left(a|\nabla u|^{2}+V_{\max } u^{2}\right) \mathrm{d} x+\frac{b}{4}\|\nabla u\|_{2}^{4}-\lambda \int_{\mathbb{R}^{3}}\left[F(u)+\frac{1}{6} u^{6}\right] \mathrm{d} x, \quad \forall u \in H^{1}\left(\mathbb{R}^{3}\right) .
$$

In view of Theorem 1.3, under (F1)-(F3), there exists $u^{\infty} \in \mathcal{M}_{1}^{\infty}$ such that

$$
\left(\mathcal{J}_{1}^{\infty}\right)^{\prime}\left(u^{\infty}\right)=0 \text { and } m_{1}^{\infty}=\mathcal{J}_{1}^{\infty}\left(u^{\infty}\right) .
$$


It is easy to check that there exists $T>1$ such that

$$
\mathrm{J}_{\lambda}^{\star}\left(t^{1 / 2}\left(u^{\infty}\right) t\right)<0, \quad \forall t \geq T .
$$

By (4.8) and simple calculation, we can derive the following lemma.

Lemma 4.3. Assume that (V1), (V2) and (F1)-(F3) hold. Then

(i) $\mathcal{J}_{\varepsilon, \lambda}\left(T^{1 / 2}\left(u^{\infty}\right)_{T}\right)<0$ for all $\lambda \in[1 / 2,1]$ and $\varepsilon \geq 0$;

(ii) there exist a constant $\kappa_{0}$ independent of $\lambda$ and $\varepsilon$ such that for all $\lambda \in[1 / 2,1]$ and $\varepsilon \geq 0$,

$$
c_{\varepsilon, \lambda}=\inf _{\gamma \in \Gamma} \max _{t \in[0,1]} \mathcal{J}_{\mathcal{E}, \lambda}(\gamma(t)) \geq \kappa_{0}>\max \left\{\mathcal{J}_{\varepsilon, \lambda}(0), \mathcal{J}_{\mathcal{E}, \lambda}\left(T^{1 / 2}\left(u^{\infty}\right)_{T}\right)\right\},
$$

where

$$
\Gamma=\left\{\gamma \in \mathcal{C}\left([0,1], H^{1}\left(\mathbb{R}^{3}\right)\right): \gamma(0)=0, \gamma(1)=T^{1 / 2}\left(u^{\infty}\right)_{T}\right\} ;
$$

(iii) $m_{\lambda}^{\infty}$ is non-increasing on $\lambda \in[1 / 2,1]$;

(iv) $c_{\varepsilon, \lambda}$ is non-increasing and left continuous on $\lambda \in[1 / 2,1]$ for $\varepsilon \geq 0$.

Since $V \in \mathcal{C}\left(\mathbb{R}^{3}, \mathbb{R}\right), V(0)<V_{\infty}$ and $u \in H^{1}\left(\mathbb{R}^{3}\right) \backslash\{0\}$, then there exist $\bar{r}>0$ and $\bar{R}>0$ such that

$$
\begin{gathered}
V_{\infty}-V(x)>\frac{1}{4}\left(V_{\infty}-V(0)\right), \quad \forall|x| \leq \bar{r}, \\
{\left[V_{\infty}-V(0)+4 \cdot 3^{4}\left(V_{\max }-V_{\infty}\right)\right] \int_{|x|>\bar{R}}\left|u^{\infty}\right|^{2} \mathrm{~d} x \leq \frac{1}{2}\left(V_{\infty}-V(0)\right)\left\|u^{\infty}\right\|_{2}^{2},}
\end{gathered}
$$

and

$$
T^{4}\left(V_{\max }-V_{\infty}\right) \int_{|x|>\bar{R}}\left|u^{\infty}\right|^{2} \mathrm{~d} x \leq \frac{\min \{\alpha(1 / 2), \alpha(3 / 2)\}}{6+p} V_{\infty}\left\|u^{\infty}\right\|_{2}^{2} .
$$

Lemma 4.4. Assume that (V1), (V2) and (F1)-(F3) hold. Then there exists $\bar{\lambda} \in[1 / 2,1]$ such that $c_{\varepsilon, \lambda}<m_{\lambda}^{\infty}$ for $\lambda \in(\bar{\lambda}, 1]$ and $\varepsilon \in\left[0, \varepsilon_{\star}\right]$, where $\varepsilon_{\star}=\bar{r} / \bar{R} T$.

Proof. For any $\varepsilon \geq 0$, it is easy to see that $\mathcal{J}_{\varepsilon, \lambda}\left(t^{1 / 2}\left(u^{\infty}\right)_{t}\right)$ is continuous on $t \in(0, \infty)$. Hence for any $\lambda \in[1 / 2,1]$ and $\varepsilon \geq 0$, there exists a $t_{\varepsilon, \lambda} \in(0, T)$ such that $\mathcal{J}_{\varepsilon, \lambda}\left(\left(t_{\varepsilon, \lambda}\right)^{1 / 2}\left(u^{\infty}\right)_{t_{\varepsilon, \lambda}}\right)=\max _{t \in(0, T]} \mathcal{J}_{\varepsilon, \lambda}\left(t^{1 / 2}\left(u^{\infty}\right)_{t}\right)$. Set

$$
\gamma_{0}(t)= \begin{cases}(t T)^{1 / 2}\left(u^{\infty}\right)_{(t T)}, & \text { for } t>0, \\ 0, & \text { for } t=0\end{cases}
$$

Then $\gamma_{0} \in \Gamma, \Gamma$ is defined in (4.10). Moreover, we have

$$
\mathcal{J}_{\varepsilon, \lambda}\left(\left(t_{\varepsilon, \lambda}\right)^{1 / 2}\left(u^{\infty}\right)_{t_{\varepsilon, \lambda}}\right)=\max _{t \in[0,1]} \mathcal{J}_{\varepsilon, \lambda}(\gamma(0)(t)) \geq c_{\varepsilon, \lambda}
$$

From (F2) and (3.22), we can deduce that the function $F(t) / t|t|^{p-1}$ is nondecreasing on $t \in(-\infty, 0) \cup(0,+\infty)$. Since $t_{\varepsilon, \lambda} \in(0, T)$, then

$$
\frac{F\left(\left(t_{\varepsilon, \lambda}\right)^{1 / 2} u^{\infty}\right)}{\left(t_{\varepsilon, \lambda}\right)^{p / 2}} \leq \frac{F\left(T^{1 / 2} u^{\infty}\right)}{T^{p / 2}}
$$

Let

$$
\begin{gathered}
\bar{\lambda}=\max \left\{\frac{1}{2}, 1-\frac{\left[V_{\infty}-V_{0}\right]\left\|u^{\infty}\right\|_{2}^{2}}{2^{8} T^{3}\left[\int_{\mathbb{R}^{3}} F\left(t^{1 / 2} u^{\infty}\right) \mathrm{d} x+T^{3}\left\|u^{\infty}\right\|_{6}^{6}\right]},\right. \\
\left.1-\frac{\min \{\alpha(1 / 2), \alpha(3 / 2)\} b\|\nabla u\|_{2}^{4}}{4(6+p) T^{3}\left[\int_{\mathbb{R}^{3}} F\left(T^{1 / 2} u^{\infty}\right) \mathrm{d} x+T^{3}\|u\|_{6}^{6}\right]}\right\} .
\end{gathered}
$$

Then $1 / 2 \leq \bar{\lambda}<1$. We have two cases to distinguish. 
Case (i) $t_{\varepsilon, \lambda} \in[1 / 2,3 / 2]$. From (4.1), (4.2), (4.6)-(4.16) and Lemma 4.3(iii), we have for all $\lambda \in(\bar{\lambda}, 1]$ and $\varepsilon \in[0, \varepsilon \star]$

$$
\begin{aligned}
m_{\lambda}^{\infty} \geq & m_{1}^{\infty}=\mathcal{J}_{1}^{\infty}\left(\left(t_{\varepsilon, \lambda}\right)^{1 / 2}\left(u^{\infty}\right)_{t_{\varepsilon, \lambda}}\right) \\
= & \mathcal{J}_{\varepsilon, \lambda}^{\infty}\left(\left(t_{\varepsilon, \lambda}\right)^{1 / 2}\left(u^{\infty}\right)_{t_{\varepsilon, \lambda}}\right)-(1-\lambda)\left(t_{\varepsilon, \lambda}\right)^{3} \int_{\mathbb{R}^{3}}\left[F\left(\left(t_{\varepsilon, \lambda}\right)^{1 / 2} u^{\infty}\right)+\left(t_{\varepsilon, \lambda}\right)^{3}\left|u^{\infty}\right|^{6}\right] \mathrm{d} x \\
& +\frac{\left(t_{\varepsilon, \lambda}\right)^{4}}{2} \int_{\mathbb{R}^{3}}\left[V_{\infty}-V\left(\varepsilon t_{\varepsilon, \lambda} x\right)\right]\left|u^{\infty}\right|^{2} \mathrm{~d} x \\
\geq & c_{\varepsilon, \lambda}-(1-\lambda) T^{3} \int_{\mathbb{R}^{3}}\left[F\left(T^{1 / 2} u^{\infty}\right)+T^{3}\left|u^{\infty}\right|^{6}\right] \mathrm{d} x \\
& +\frac{V_{\infty}-V(0)}{2^{7}} \int_{|x| \leq \bar{R}}\left|u^{\infty}\right|^{2} \mathrm{~d} x-\frac{3^{4}\left(V_{\max }-V_{\infty}\right)}{2^{5}} \int_{|x|>\bar{R}}\left|u^{\infty}\right|^{2} \mathrm{~d} x \\
\geq & c_{\varepsilon, \lambda}-(1-\lambda) T^{3} \int_{\mathbb{R}^{3}}\left[F\left(T^{1 / 2} u^{\infty}\right)+T^{3}\left|u^{\infty}\right|^{6}\right] \mathrm{d} x+\frac{V_{\infty}-V(0)}{2^{7}}\left\|u^{\infty}\right\|_{2}^{2} \\
& -\frac{V_{\infty}-V(0)+4 \cdot 3^{4}\left(V_{\max }-V_{\infty}\right)}{2^{7}} \int_{|x|>\bar{R}}\left|u^{\infty}\right|^{2} \mathrm{~d} x \\
> & c_{\varepsilon, \lambda} .
\end{aligned}
$$

Case (ii) $t_{\varepsilon, \lambda} \in(0,1 / 2) \cup(3 / 2, T]$. From (4.1), (4.2), (4.11), (4.13)-(4.16) and Lemma 4.3(iii), we have

$$
\begin{aligned}
& m_{\lambda}^{\infty} \geq=m_{1}^{\infty}=\mathcal{J}_{1}^{\infty}\left(u^{\infty}\right) \geq \mathcal{J}_{1}^{\infty}\left(\left(t_{\varepsilon, \lambda}\right)^{1 / 2}\left(u^{\infty}\right)_{t_{\varepsilon, \lambda}}\right)+\frac{\alpha\left(t_{\varepsilon, \lambda}\right)}{4(6+p)}\left(2 V_{\infty}\left\|u^{\infty}\right\|_{2}^{2}+b\left\|\nabla u^{\infty}\right\|_{2}^{4}\right) \\
&= \mathcal{J}_{\varepsilon, \lambda}^{\infty}\left(\left(t_{\varepsilon, \lambda}\right)^{1 / 2}\left(u^{\infty}\right)_{t_{\varepsilon, \lambda}}\right)-(1-\lambda)\left(t_{\varepsilon, \lambda}\right)^{3} \int_{\mathbb{R}^{3}}\left[F\left(\left(t_{\varepsilon, \lambda}\right)^{1 / 2} u^{\infty}\right)+\left(t_{\varepsilon, \lambda}\right)^{3}\left|u^{\infty}\right|^{6}\right] \mathrm{d} x \\
&+\frac{\left(t_{\varepsilon, \lambda}\right)^{4}}{2} \int_{\mathbb{R}^{3}}\left[V_{\infty}-V\left(\varepsilon t_{\varepsilon, \lambda} x\right)\right]\left|u^{\infty}\right|^{2} \mathrm{~d} x+\frac{\alpha\left(t_{\varepsilon, \lambda}\right)}{4(6+p)}\left(2 V_{\infty}\left\|u^{\infty}\right\|_{2}^{2}+b\left\|\nabla u^{\infty}\right\|_{2}^{4}\right) \\
& \geq c_{\varepsilon, \lambda}-(1-\lambda) T^{3} \int_{\mathbb{R}^{3}}\left[F\left(T^{1 / 2} u^{\infty}\right)+T^{3}\left|u^{\infty}\right|^{6}\right] \mathrm{d} x-\frac{T^{4}\left(V_{\max }-V_{\infty}\right)}{2} \int_{|x|>\bar{R}}\left|u^{\infty}\right|^{2} \mathrm{~d} x \\
& \\
& \quad+\frac{\min \{\alpha(1 / 2), \alpha(3 / 2)\}}{4(6+p)}\left(2 V_{\infty}\left\|u^{\infty}\right\|_{2}^{2}+b\|\nabla u\|_{2}^{4}\right) \\
&>c_{\varepsilon, \lambda} \text { for all } \lambda \in(\lambda *, 1], \varepsilon \in\left[0, \varepsilon_{\star}\right] .
\end{aligned}
$$

In both cases, we conclude that $c_{\varepsilon, \lambda}<m_{\lambda}^{\infty}$ for all $\lambda \in\left(\lambda_{\star}, 1\right]$ and $\varepsilon \in\left[0, \varepsilon_{\star}\right]$.

Similarly to the proofs of Lemma 3.3 and 3.4, we have that $m_{\lambda}^{\infty}<\frac{a S}{3}\left(\frac{b S^{2}+\sqrt{b^{2} S^{4}+4 \lambda a S}}{2 \lambda}\right)+$ $\frac{b S^{2}}{12}\left(\frac{b S^{2}+\sqrt{b^{2} S^{4}+4 \lambda a S}}{2 \lambda}\right)^{2}$ under the assumption of Theorem 1.1. Then, analogous to the proof of Lemma 3.4 in [22], we can obtain the following lemma.

Lemma 4.5. Assume that (V1), (V2) and (F1)-(F3) hold. Let $\left\{u_{n}\right\}$ be a bounded (PS) sequence of $c_{\varepsilon, \lambda}$ for $\mathcal{J}_{\varepsilon, \lambda}$ with $\lambda \in[1 / 2,1]$. Then there exists a subsequence of $\left\{u_{n}\right\}$, still denoted by $\left\{u_{n}\right\}$, and $u_{0} \in H^{1}\left(\mathbb{R}^{3}\right)$ such that $A^{2}:=\lim _{n \rightarrow \infty}\left\|\nabla u_{n}\right\|$ exist, $u_{n} \rightarrow u_{0}$ in $H^{1}\left(\mathbb{R}^{3}\right)$ and $G_{\varepsilon, \lambda}^{\prime}\left(u_{0}\right)=0$, where

$$
G_{\varepsilon, \lambda}(u)=\frac{a+b A^{2}}{2} \int_{\mathbb{R}^{3}}|\nabla u|^{2} \mathrm{~d} x+\frac{1}{2} \int_{\mathbb{R}^{3}} V(\varepsilon x) u^{2} \mathrm{~d} x-\lambda \int_{\mathbb{R}^{3}}\left[F(u)+\frac{1}{6} u^{6}\right] \mathrm{d} x
$$

and either 
(i) $u_{n} \rightarrow u_{0}$ in $H^{1}\left(\mathbb{R}^{3}\right)$; or

(ii) there exist an integer $l \in \mathbb{N}$ and $w^{1}, \ldots, w^{l} \in H^{1}\left(\mathbb{R}^{3}\right) \backslash\{0\}$ such that $\left(G_{\lambda}^{\infty}\right)^{\prime}\left(w^{k}\right)=0$, for $1 \leq k \leq l$, and

$$
\begin{gathered}
c_{\varepsilon, \lambda}+\frac{b A^{4}}{4}=G_{\varepsilon, \lambda}\left(u_{0}\right)+\sum_{k=1}^{l} G_{\lambda}^{\infty}\left(w^{k}\right) ; \\
A^{2}=\left\|\nabla u_{0}\right\|_{2}^{2}+\sum_{k=1}^{l}\left\|\nabla w^{k}\right\|_{2}^{2},
\end{gathered}
$$

where

$$
G_{\lambda}^{\infty}(u)=\frac{a+b A^{2}}{2} \int_{\mathbb{R}^{3}}|\nabla u|^{2} \mathrm{~d} x+\frac{V_{\infty}}{2} \int_{\mathbb{R}^{3}} u^{2} \mathrm{~d} x-\lambda \int_{\mathbb{R}^{3}}\left[F(u)+\frac{1}{6} u^{6}\right] \mathrm{d} x .
$$

Lemma 4.6. Assume that (V1), (V2) and (F1)-(F3) hold. Then for every $\varepsilon \in\left[0, \varepsilon^{\star}\right]$ and for almost every $\lambda \in$ $(\bar{\lambda}, 1]$, there exists $u_{\varepsilon, \lambda} \in H^{1}\left(\mathbb{R}^{3}\right) \backslash\{0\}$ such that

$$
\left(\mathcal{J}_{\varepsilon, \lambda}\right)^{\prime}\left(u_{\varepsilon, \lambda}\right)=0, \quad \mathcal{J}_{\varepsilon, \lambda}\left(u_{\varepsilon, \lambda}\right)=c_{\varepsilon, \lambda} .
$$

Proof. By [19, Theorem 1.1], we have that for almost every $\lambda \in[1 / 2,1]$, and for every $\varepsilon \in\left[0, \varepsilon_{\star}\right]$, there exist a bounded sequence $\left\{u_{n}(\lambda, \varepsilon)\right\} \subset H^{1}\left(\mathbb{R}^{3}\right)$ denoted by $\left\{u_{n}\right\}$ for simplicity, such that

$$
\mathcal{J}_{\varepsilon, \lambda}\left(u_{n}\right) \rightarrow c_{\varepsilon, \lambda}>0,\left\|\mathcal{J}_{\mathcal{E}, \lambda}^{\prime}\left(u_{n}\right)\right\| \rightarrow 0, \text { as } n \rightarrow \infty .
$$

From Lemma 4.5, it can be deduced easily that there exists a subsequence of $\left\{u_{n}\right\}$, still denoted by $\left\{u_{n}\right\}$, and $u_{\varepsilon, \lambda} \in H^{1}\left(\mathbb{R}^{3}\right)$ such that $A_{\varepsilon, \lambda}^{2}=\lim _{n \rightarrow \infty}\left\|\nabla u_{\varepsilon, \lambda}\right\|$ exists, $u_{n} \rightarrow u_{\varepsilon, \lambda}$ in $H^{1}\left(\mathbb{R}^{3}\right)$ and $\left(H_{\varepsilon, \lambda}\right)^{\prime}\left(u_{\varepsilon, \lambda}\right)=0$, and either (i) or (ii) occurs, where

$$
H_{\varepsilon, \lambda}(u)=\frac{a+b A^{2}}{2} \int_{\mathbb{R}^{3}}|\nabla u|^{2} \mathrm{~d} x+\frac{1}{2} \int_{\mathbb{R}^{3}} V(\varepsilon x) u^{2} \mathrm{~d} x-\lambda \int_{\mathbb{R}^{3}}\left[F(u)+\frac{1}{6} u^{6}\right] \mathrm{d} x .
$$

If (ii) occurs, i.e. there exist $l \in \mathbb{N}$ and $w^{1}, \ldots, w^{l}$ in $H^{1}\left(\mathbb{R}^{3}\right) \backslash\{0\}$ such that $\left(H_{\lambda}^{\infty}\right)^{\prime}\left(w^{k}\right)=0$ for $1 \leq k \leq l$,

$$
c_{\varepsilon, \lambda}+\frac{b A_{\varepsilon, \lambda}^{4}}{4}=G_{\varepsilon, \lambda}\left(u_{\varepsilon, \lambda}\right)+\sum_{k=1}^{l} G_{\lambda}^{\infty}\left(w^{k}\right)
$$

and

$$
A_{\varepsilon, \lambda}^{2}=\left\|\nabla u_{\varepsilon, \lambda}\right\|_{2}^{2}+\sum_{k=1}^{l}\left\|\nabla w^{k}\right\|_{2}^{2}
$$

where

$$
H_{\lambda}^{\infty}(u)=\frac{a+b A_{\varepsilon, \lambda}^{2}}{2} \int_{\mathbb{R}^{3}}|\nabla u|^{2} \mathrm{~d} x+\frac{V_{\infty}}{2} \int_{\mathbb{R}^{3}} u^{2} \mathrm{~d} x-\lambda \int_{\mathbb{R}^{3}}\left[F(u)+\frac{1}{6} u^{6}\right] \mathrm{d} x .
$$

Since $\left(H_{\varepsilon, \lambda}\right)^{\prime}\left(u_{\varepsilon, \lambda}\right)=0$, then we have the Pohožaev identity of the functional $H_{\varepsilon, \lambda}$

$$
\begin{aligned}
\hat{\mathcal{P}}_{\varepsilon, \lambda}\left(u_{\varepsilon, \lambda}\right)= & \frac{a+b A_{\varepsilon, \lambda}^{2}}{2}\left\|\nabla u_{\varepsilon, \lambda}\right\|_{2}^{2}+\frac{1}{2} \int_{\mathbb{R}^{3}}[3 V(\varepsilon x)+\nabla V(\varepsilon x) \cdot(\varepsilon x)] u_{\varepsilon, \lambda}^{2} \mathrm{~d} x \\
& +\frac{b}{2}\left\|\nabla u_{\varepsilon, \lambda}\right\|_{2}^{4}-3 \lambda \int_{\mathbb{R}^{3}}\left[F\left(u_{\varepsilon, \lambda}\right)+\frac{1}{6} u_{\varepsilon, \lambda}^{6}\right] \mathrm{d} x .
\end{aligned}
$$

It follows from (3.22), (4.21) and (4.24), that

$$
\begin{aligned}
H_{\varepsilon, \lambda}\left(u_{\varepsilon, \lambda}\right) & =H_{\varepsilon, \lambda}\left(u_{\varepsilon, \lambda}\right)-\frac{2}{6+p}\left[\frac{1}{2}\left\langle\left(H_{\varepsilon, \lambda}\right)^{\prime}\left(u_{\varepsilon, \lambda}\right), u_{\varepsilon, \lambda}\right\rangle-\hat{\mathcal{P}}_{\varepsilon, \lambda}\left(u_{\varepsilon, \lambda}\right)\right] \\
& \geq\left(\frac{1}{2}-\frac{2}{6+p}\right) b A_{\varepsilon, \lambda}^{2}\left\|\nabla u_{\varepsilon, \lambda}\right\|_{2}^{2} .
\end{aligned}
$$


Since $\left(H_{\lambda}^{\infty}\right)^{\prime}\left(w^{k}\right)=0$, then we have the Pohožaev identity of the functional $H_{\lambda}^{\infty}$

$$
\begin{aligned}
\hat{\mathcal{P}}_{\lambda}^{\infty}\left(w^{k}\right)= & \frac{a+b A_{\varepsilon, \lambda}^{2}}{2}\left\|\nabla w^{k}\right\|_{2}^{2}+\frac{3}{2} \int_{\mathbb{R}^{3}} V_{\infty}\left(w^{k}\right)^{2} \mathrm{~d} x \\
& +\frac{b}{2}\left\|\nabla w^{k}\right\|_{2}^{4}-3 \lambda \int_{\mathbb{R}^{3}}\left[F\left(w^{k}\right)+\frac{1}{6}\left(w^{k}\right)^{6}\right] \mathrm{d} x .
\end{aligned}
$$

Then, from (4.4), (4.23), (4.24) and (4.26), we have

$$
\begin{aligned}
0 & =\frac{1}{2}\left\langle\left(H_{\lambda}^{\infty}\right)^{\prime} w^{k}, w^{k}\right\rangle+\hat{\mathcal{P}}_{\lambda}^{\infty}\left(w^{k}\right) \\
& \geq \mathcal{J}_{\lambda}^{\infty}\left(w^{k}\right) .
\end{aligned}
$$

Since $w^{k} \in H^{1}\left(\mathbb{R}^{3}\right)$, in view of Lemma 2.3, there exists $t_{k}>0$ such that $t_{k}^{1 / 2}\left(w^{k}\right)_{t^{k}} \in \mathcal{M}_{\lambda}^{\infty}$. From (4.2), (4.4), (4.6), (4.24) and (4.27), one has

$$
\begin{aligned}
H_{\lambda}^{\infty}\left(w^{k}\right)= & H_{\lambda}^{\infty}\left(w^{k}\right)-\frac{2}{6+p}\left[\frac{1}{2}\left\langle\left(H_{\lambda}^{\infty}\right)^{\prime} w^{k}, w^{k}\right\rangle+\hat{\mathcal{P}}_{\lambda}^{\infty}\left(w^{k}\right)\right] \\
= & \left(\frac{1}{2}-\frac{2}{6+p}\right)\left(a+b A^{2}\right)\left\|\nabla w^{k}\right\|_{2}^{2}+\frac{p-2}{6+p} \int_{\mathbb{R}^{3}} V_{\infty}\left|w^{k}\right|^{2} \mathrm{~d} x \\
& +\frac{2 \lambda}{6+p} \int_{\mathbb{R}^{3}}\left[f\left(w^{k}\right) w^{k}-p F\left(w^{k}\right)\right] \mathrm{d} x+\frac{(1-p) \lambda}{6(6+p)} \int_{\mathbb{R}^{3}}\left|w^{k}\right|^{6} \mathrm{~d} x \\
= & \frac{1}{4} b A_{\varepsilon, \lambda}^{2}\left\|\nabla w^{k}\right\|_{2}^{2}+\mathcal{J}_{\lambda}^{\infty}\left(w^{k}\right)-\frac{2}{6+p} \partial_{\lambda}^{\infty}\left(w^{k}\right) \\
\geq & \frac{1}{4} b A_{\varepsilon, \lambda}^{2}\left\|\nabla w^{k}\right\|_{2}^{2}+\mathcal{J}_{\lambda}^{\infty}\left(t_{k}^{1 / 2}\left(w^{k}\right)_{t_{k}}\right)-\frac{2 t_{k}^{6+p} / 2}{6+p} \partial_{\lambda}^{\infty}\left(w^{k}\right) \\
\geq & \frac{1}{4} b A_{\varepsilon, \lambda}^{2}\left\|\nabla w^{k}\right\|_{2}^{2}+m_{\lambda}^{\infty} .
\end{aligned}
$$

It follows from (4.22), (4.23), (4.25) and (4.28) that

$$
\begin{aligned}
c_{\varepsilon, \lambda}+\frac{b A_{\varepsilon, \lambda}^{4}}{4} & =H_{\varepsilon, \lambda}\left(u_{\varepsilon, \lambda}\right)+\sum_{k=1}^{l} H_{\lambda}^{\infty}\left(w^{k}\right) \\
& \geq l m_{\lambda}^{\infty}+\frac{1}{4} b A_{\varepsilon, \lambda}^{4}\left[\left\|\nabla u_{\varepsilon, \lambda}\right\|_{2}^{2}+\sum_{k=1}^{l}\left\|w^{k}\right\|_{2}^{2}\right] \\
& \geq m_{\lambda}^{\infty}+\frac{b A_{\varepsilon, \lambda}^{4}}{4}, \quad \forall \lambda \in(\bar{\lambda}, 1], \quad \forall \varepsilon \in\left[0, \varepsilon_{\star}\right] .
\end{aligned}
$$

It contradicts with Lemma 4.4. Thus $u_{n} \rightarrow u_{\varepsilon, \lambda}$ in $H^{1}\left(\mathbb{R}^{3}\right)$ and $\mathcal{J}_{\varepsilon, \lambda}\left(u_{\varepsilon, \lambda}\right)=c_{\varepsilon, \lambda}$.

Proof of Theorem 1.1. In view of Lemma 4.6, for any fixed $\varepsilon \in\left[0, \varepsilon_{\star}\right]$, there exist two sequences $\left\{\lambda_{n}\right\} \subset[\bar{\lambda}, 1]$ and $\left\{u_{\varepsilon, \lambda_{n}}\right\} \subset H^{1}\left(\mathbb{R}^{3}\right) \backslash\{0\}$, denoted by $\left\{u_{n}\right\}$, such that

$$
\lambda_{n} \rightarrow 1, \quad\left(\mathcal{J}_{\varepsilon, \lambda_{n}}\right)^{\prime}\left(u_{n}\right)=0, \mathcal{J}_{\mathcal{E}, \lambda_{n}}\left(u_{n}\right)=c_{\varepsilon, \lambda} .
$$

Then, it follows from (V2), (3.26), (4.1), and Lemma 4.3 (iv), that

$$
c_{\varepsilon, 1}+o(1)=c_{\varepsilon, \lambda_{n}}=\mathcal{J}_{\varepsilon, \lambda_{n}}\left(u_{n}\right)-\frac{2}{6+p} \mathcal{J}_{\varepsilon, \lambda_{n}}\left(u_{n}\right) \geq \frac{p+2}{2(6+p)} a\left\|\nabla u_{n}\right\|_{2}^{2} .
$$

Since $\left\langle\mathcal{J}_{\varepsilon, \lambda_{n}}^{\prime}\left(u_{n}\right), u_{n}\right\rangle=0$, from (4.29), (4.30) and the Sobolev embedding inequality, we can deduce that $\left\{u_{n}\right\}$ in bounded in $H^{1}\left(\mathbb{R}^{3}\right)$. Since $c_{\varepsilon, \lambda_{n}} \rightarrow c_{\varepsilon, 1}$, similar to the proof of Lemma 4.6, for any fixed $\varepsilon \in\left[0, \varepsilon_{\star}\right]$, we derive that there exist $\hat{u}_{\varepsilon} \in H^{1}\left(\mathbb{R}^{3}\right) \backslash\{0\}$ such that

$$
\mathcal{J}_{\varepsilon}^{\prime}\left(\hat{u}_{\varepsilon}\right)=0, \quad \mathcal{J}_{\varepsilon}\left(\hat{u}_{\varepsilon}\right)=c_{\varepsilon, 1}>0 .
$$


For $\varepsilon \in[0, \varepsilon \star]$, set

$$
\mathcal{K}_{\varepsilon}=\left\{u \in H^{1}\left(\mathbb{R}^{3}\right) \backslash\{0\}: \mathcal{J}_{\varepsilon}^{\prime}(u)=0\right\}, \quad m_{\varepsilon}^{\star}=\inf _{u \in \mathcal{K}_{\varepsilon}} \mathcal{J}_{\varepsilon}(u) .
$$

Then (4.31) shows that $\mathcal{K}_{\varepsilon} \neq \varnothing$ and $m_{\varepsilon}^{\star} \leq c_{\varepsilon, 1}$. Since $\mathcal{K}_{\varepsilon} \subset \mathcal{M}_{\varepsilon}$ and Lemma 4.4, we also have $0<m_{\varepsilon} \leq m_{\varepsilon}^{\star} \leq$ $c_{\varepsilon, 1}<m_{1}^{\infty}$. Let $\left\{u_{n}\right\} \subset \mathcal{K}_{\varepsilon}$ be such that $\mathcal{J}_{\varepsilon}^{\prime}\left(u_{n}\right)=0$ and $\mathcal{J}_{\varepsilon}\left(u_{n}\right) \rightarrow m_{\varepsilon}^{\star}$. Arguing as the proof of Lemma 4.6, we can prove that there exists $u_{\varepsilon}^{*} \in H^{1}\left(\mathbb{R}^{3}\right) \backslash\{0\}$ such that $\mathcal{J}_{\varepsilon}\left(u_{\varepsilon}^{*}\right)=m_{\varepsilon}^{*}$ and $\mathcal{J}_{\varepsilon}^{\prime}\left(u_{\varepsilon}^{*}\right)=0$. This shows that $u_{\varepsilon}^{*}$ is a ground state solution of $\left(P K_{\varepsilon}\right)$ for every $\varepsilon \in\left[0, \varepsilon^{*}\right]$. Hence, the function $v_{\varepsilon}^{\star}=u_{\varepsilon}^{\star}(x / \varepsilon)$ is a ground state solution of $\left(S K_{\varepsilon}\right)$ for every $\varepsilon \in\left[0, \varepsilon_{\star}\right]$.

\section{Ground state solutions of Nehari-pohožaev type}

In this section, we consider the existence of ground state solution of $\left(P K_{\varepsilon}\right)$. By (1.1), (1.2), (1.4), (1.5), (1.7), (1.8) and (1.9), one has $\mathcal{J}_{0}=I_{V_{0}}, \partial_{0}=J_{V_{0}}$ and $\mathcal{M}_{0}=\hat{\mathcal{M}}_{V_{0}}$. Let

$$
\hat{V}=\frac{1}{2}\left(V_{\infty}+V_{0}\right)=\frac{1}{2}\left(V_{\infty}+V(0)\right) \text {. }
$$

Applying Theorem 1.3, there exist $\hat{u}_{0} \in \hat{\mathcal{M}}_{V_{0}}$ and $\hat{u} \in \mathcal{M}_{\hat{V}}$ such that

$$
\mathcal{J}_{0}^{\prime}\left(\hat{u}_{0}\right)=0, \quad \mathcal{J}_{0}\left(\hat{u}_{0}\right)=m_{0}=\inf _{u \in \mathcal{M}_{0}} \mathcal{J}_{0}(u)=\inf _{u \in H^{1}\left(\mathbb{R}^{3}\right) \backslash\{0\}} \max _{t>0} \mathcal{J}_{0}\left(t^{1 / 2}(u)_{t}\right)>0
$$

and

$$
I_{\hat{V}}^{\prime}(\hat{u})=0, \quad I_{\hat{V}}(\hat{u})=\hat{m}_{\hat{V}}=\inf _{u \in \hat{\mathcal{M}}_{\hat{V}}} I_{\hat{V}}(u)=\inf _{u \in H^{1}\left(\mathbb{R}^{3}\right) \backslash\{0\}} \max _{t>0} I_{\hat{V}}\left(t^{1 / 2}(u)_{t}\right)>0 .
$$

In view of Lemma 2.3, there exists $t_{0}>0$ such that

$$
t_{0}^{1 / 2}(\hat{u})_{t_{0}} \in \mathcal{M}_{0}, \quad J_{0}\left(t_{0}^{1 / 2}(\hat{u})_{t_{0}}\right) \geq m_{0} .
$$

Let

$$
I_{\star}(u)=\frac{1}{2} \int_{\mathbb{R}^{3}}\left(a|\nabla u|^{2}+V_{\max } u^{2}\right) \mathrm{d} x+\frac{b}{4}\|\nabla u\|_{2}^{4}-\int_{\mathbb{R}^{3}}\left[F(u)+\frac{1}{6} u^{6}\right] \mathrm{d} x, \forall u \in H^{1}\left(\mathbb{R}^{3}\right) .
$$

Using (F1), (F2) and (4.8), it is easy to check that there exists $T_{0}>1$ such that

$$
I \star\left(t^{1 / 2}(u)_{t}\right)<0, \quad \forall t>T_{0} .
$$

In view of (5.6) and Lemma 2.3, for any $\varepsilon>0$, there exists $t_{\varepsilon} \in\left(0, T_{0}\right)$ such that

$$
t_{\varepsilon}^{1 / 2}\left(\hat{u}_{0}\right)_{t_{\varepsilon}} \in \mathcal{M}_{\varepsilon}, \quad \mathcal{J}_{\varepsilon}\left(t_{\varepsilon}^{1 / 2}\left(\hat{u}_{0}\right)_{t_{\varepsilon}}\right) \geq m_{\varepsilon} .
$$

Lemma 5.1. Assume that (V1), (V3) and (F1)-(F3) hold. Then $\hat{m}_{\hat{V}} \geq m_{0}+\delta_{0}$, where

$$
\delta_{0}=\frac{V_{\infty}-V_{0}}{4} t_{0}^{4}\|\hat{u}\|_{2}^{2}>0
$$

is independent of $\varepsilon>0$.

Proof. By (5.3) and (5.4),

$$
\begin{aligned}
\hat{m}_{\hat{V}}=I_{\hat{V}}(\hat{u}) \geq I_{\hat{V}}\left(t_{0}^{1 / 2}(\hat{u})_{t_{0}}\right) & =\mathcal{J}_{0}\left(t_{0}^{1 / 2}(\hat{u})_{t_{0}}\right)+\frac{\hat{V}-V_{0}}{2} t_{0}^{4} \int_{\mathbb{R}^{3}}|\hat{u}|^{2} \mathrm{~d} x \\
& \geq m_{0}+\frac{V_{\infty}-V_{0}}{4} t_{0}^{4} \int_{\mathbb{R}^{3}}|\hat{u}|^{2} \mathrm{~d} x \\
& =m_{0}+\delta_{0} .
\end{aligned}
$$


Now we choose $R_{0}>0$ large such that

$$
V(x) \geq \hat{V}, \quad\left[1+T_{0}^{(6+p) / 2}\right] V_{\max } \int_{|x| \geq R_{0}} \hat{u}_{0} \mathrm{~d} x \leq \delta_{0}, \quad \forall|x|>R_{0} .
$$

By (V1) and (V3), there exists $\varepsilon_{0}>0$ small enough such that

$$
\begin{aligned}
& \left\|\hat{u}_{0}\right\|_{2}^{2} \sup _{|x| \leq R_{0}}\left\{\left[p-2+8 T_{0}^{(6+p) / 2}\right]|V(\varepsilon x)-V(0)|\right. \\
& \left.\quad+2\left[1+T_{0}^{(6+p) / 2}\right]|\nabla V(\varepsilon x) \cdot(\varepsilon x)|\right\} \leq \frac{\delta_{0}(6+p)}{2}, \forall \varepsilon \in\left[0, \varepsilon_{0}\right] .
\end{aligned}
$$

Lemma 5.2. Assume that (V1), (V3) and (F1)-(F3) hold. Then $m_{0} \geq m_{\varepsilon}-3 \delta_{0} / 4$ for every $\varepsilon \in\left[0, \varepsilon_{0}\right]$.

Proof. By (2.1), we have $h_{1}(0, y) \geq 0$ for all $y \in \mathbb{R}^{3}$, which, together with (2.12) implies

$$
-4 V(y) \leq \nabla V(y) \cdot y \leq \frac{p-2}{2} V(y), \quad \forall y \in \mathbb{R}^{3} .
$$

Since $\mathcal{J}_{0}\left(\hat{u}_{0}\right)=0$ by (5.2), it follows from (1.1), (1.4), (2.3), (5.7), (5.9), (5.10) and (5.11) that

$$
\begin{aligned}
& m_{0}=\mathcal{J}_{0}\left(\hat{u}_{0}\right)=\mathcal{J}_{\varepsilon}\left(\hat{u}_{\varepsilon}\right)+\frac{1}{2} \int_{\mathbb{R}^{3}}[V(0)-V(\varepsilon x)] \hat{u}_{0}^{2} \mathrm{~d} x \\
& \geq \mathcal{J}_{\varepsilon}\left(t_{\varepsilon}^{1 / 2}\left(\hat{u}_{0}\right)_{t_{\varepsilon}}\right)+\frac{2\left(1-t_{\varepsilon}^{(6+p) / 2}\right)}{2} \partial_{\varepsilon}\left(\hat{u}_{0}\right)+\frac{1}{2} \int_{\mathbb{R}^{3}}[V(0)-V(\varepsilon x)] \hat{u}_{0}^{2} \mathrm{~d} x \\
& =\mathcal{J}_{\varepsilon}\left(t_{\varepsilon}^{1 / 2}\left(\hat{u}_{0}\right)_{t_{\varepsilon}}\right)+\frac{2\left(1-t_{\varepsilon}^{(6+p) / 2}\right)}{2} \partial_{0}\left(\hat{u}_{0}\right)+\frac{1}{2} \int_{\mathbb{R}^{3}}[V(0)-V(\varepsilon x)] \hat{u}_{0}^{2} \mathrm{~d} x \\
& +\frac{1-t_{\varepsilon}^{(6+p) / 2}}{6+p} \int_{\mathbb{R}^{3}}[4 V(\varepsilon x)+\nabla V(\varepsilon x) \cdot(\varepsilon x)-4 V(0)] \hat{u}_{0}^{2} \mathrm{~d} x \\
& =\mathcal{J}_{\varepsilon}\left(t_{\varepsilon}^{1 / 2}\left(\hat{u}_{0}\right)_{t_{\varepsilon}}\right)+\frac{1}{2(6+p)} \int_{\mathbb{R}^{3}}[(p-2) V(0)-(p-2) V(\varepsilon x)+2 \nabla V(\varepsilon x) \cdot(\varepsilon x)] \hat{u}_{0}^{2} \mathrm{~d} x \\
& -\frac{t_{\varepsilon}^{(6+p) / 2}}{6+p} \int_{\mathbb{R}^{3}}[4 V(\varepsilon x)+\nabla V(\varepsilon x) \cdot(\varepsilon x)-4 V(0)] \hat{u}_{0}^{2} \mathrm{~d} x \\
& \geq m_{\varepsilon}-\frac{1}{2(6+p)} \int_{|x| \leq R_{0}}\left\{\left[p-2+8 T_{0}^{(6+p) / 2}\right]|V(\varepsilon \chi)-V(0)|\right. \\
& \left.+2\left[1+T_{0}^{(6+p) / 2}|\nabla V(\varepsilon x) \cdot(\varepsilon x)|\right]\right\} \hat{u}_{0}^{2} \mathrm{~d} x-\frac{1+T_{0}^{(6+p) / 2}}{2} V_{\max } \int_{|x|>R_{0}} \hat{u}_{0} \mathrm{~d} x \\
& \geq m_{\varepsilon}-\frac{3 \delta_{0}}{4}, \quad \forall \varepsilon \in\left[0, \varepsilon_{0}\right] .
\end{aligned}
$$

Lemma 5.3. Assume that (V1), (V3) and (F1)-(F3) hold. Then $m_{\varepsilon}$ is achieved for $\varepsilon \in\left(0, \varepsilon_{0}\right]$.

Proof. In view of Lemma 2.3 and Lemma 2.6, we have that $\mathcal{M}_{\varepsilon} \neq \varnothing$ and $m_{\varepsilon}>0$ for $\varepsilon \in\left(0, \varepsilon_{0}\right]$. For any fixed $\varepsilon \in\left(0, \varepsilon_{0}\right]$, let $\left\{u_{n}\right\} \subset \mathcal{M}_{\varepsilon}$ be such that $\mathcal{J}_{\varepsilon}\left(u_{n}\right) \rightarrow m_{\varepsilon}$. Since $\mathcal{J}_{\varepsilon}\left(u_{n}\right)=0$, then it follows from (2.9) that

$$
m_{\varepsilon}+o(1)=\mathcal{J}_{\varepsilon}\left(u_{n}\right) \geq \frac{b(p-2)}{4(6+p)}\left\|\nabla u_{n}\right\|_{2}^{4},
$$

which implies $\left\{u_{n}\right\}$ is bounded in $H^{1}\left(\mathbb{R}^{3}\right)$ together with (2.11). Passing to a subsequence, we can assume that $u_{n} \rightarrow \bar{u}$ in $H^{1}\left(\mathbb{R}^{3}\right), u_{n} \rightarrow \bar{u}$ in $L_{l o c}^{s}\left(\mathbb{R}^{3}\right)$ for all $s$, with $2 \leq s<6$ and $u_{n} \rightarrow \bar{u}$ a.e. on $\mathbb{R}^{3}$. 
Next, we claim that $\bar{u} \neq 0$. Arguing by contradiction, suppose that $\bar{u}=0$, then $u_{n} \rightarrow 0$ in $L_{\text {loc }}^{S}\left(\mathbb{R}^{3}\right)$ for $2 \leq s<6$ and $u_{n} \rightarrow 0$ a.e. on $\mathbb{R}^{3}$. In view of Lemma 2.3, there exists $t_{n}>0$ such that $t_{n}^{1 / 2}\left(u_{n}\right)_{t_{n}} \in \hat{\mathcal{M}}_{\hat{V}}$ for every $n \in \mathbb{N}$. We claim that there exist two positive constant $T_{1}<T_{2}$ such that

$$
T_{1} \leq t_{n} \leq T_{2}, \quad \forall n \in \mathbb{R} .
$$

If $t_{n} \rightarrow 0$, then it follows from (2.5), (2.10), lemma 2.6 and the boundedness of $\left\{\left\|u_{n}\right\|\right\}$ that

$$
\begin{aligned}
& 0<\hat{m}_{\hat{V}} \leq \mathcal{J}_{\hat{V}}\left(t_{n}^{1 / 2}\left(u_{n}\right)_{t_{n}}\right) \\
&= \frac{a}{2} t_{n}^{2}\left\|\nabla u_{n}\right\|_{2}^{2}+\frac{\hat{V}}{2} t_{n}^{4}\left\|u_{n}\right\|_{2}^{2}+\frac{b}{4} t_{n}^{4}\|\nabla u\|_{2}^{4} \\
&-t_{n}^{3} \int_{\mathbb{R}^{3}} F\left(t_{n}^{1 / 2} u_{n}\right) \mathrm{d} x-t_{n}^{6}\left\|u_{n}\right\|_{6}^{6} \\
&= o(1),
\end{aligned}
$$

which is impossible. Hence, the first inequality holds in (5.12). On the other hand, it is easy to see that

$$
\liminf _{n \rightarrow \infty}\left\|u_{n}\right\|_{6}>0 \text {. }
$$

If not, then there exists a subsequence $\left\{u_{n_{k}}\right\}$ of $\left\{u_{n}\right\}$ such that $u_{n_{k}} \rightarrow 0$ in $L^{6}\left(\mathbb{R}^{3}\right)$, and the Sobolev embedding theorem implies that $u_{n_{k}} \rightarrow 0$ in $L^{s}\left(\mathbb{R}^{3}\right)$ for all $s \in(2,6]$. By (F1), for every $\varepsilon>0$ and $q \in(2,6)$ there exists $C_{\varepsilon}>0$ such that

$$
|f(\tau) \tau|+|F(\tau)| \leq \varepsilon\left(\tau^{2}+\tau^{6}\right)+C_{\varepsilon}|\tau|^{q} \text { for all } \tau \in \mathbb{R} .
$$

Thus, as $k \rightarrow \infty$, by (2.14),

$$
\begin{aligned}
0=\partial_{\varepsilon}\left(u_{n_{k}}\right)= & a\left\|\nabla u_{n_{k}}\right\|_{2}^{2}+\frac{1}{2} \int_{\mathbb{R}^{3}}[4 V(\varepsilon x)+\nabla V(\varepsilon x) \cdot(\varepsilon x)] u_{n_{k}}^{2} \\
& +b\left\|u_{n_{k}}\right\|_{2}^{4}-\frac{1}{2} \int_{\mathbb{R}^{3}}\left[f\left(u_{n_{k}}\right) u_{n_{k}}+F\left(u_{n_{k}}\right)\right] \mathrm{d} x-\int_{\mathbb{R}^{3}}\left|u_{n_{k}}\right|^{6} \mathrm{~d} x \\
& \geq \frac{\gamma_{1}}{2}\left\|u_{n_{k}}\right\|^{2}+b\left\|\nabla u_{n_{k}}\right\|_{2}^{4}-\frac{\gamma_{1}}{4}\left(\left\|u_{n_{k}}\right\|_{2}^{2}+\left\|u_{n_{k}}\right\|_{6}^{6}\right)-C_{4}\left\|u_{n_{k}}\right\|_{q}^{q}-\left\|u_{n_{k}}\right\|_{6}^{6} \\
& \geq \frac{\gamma_{1}}{4}\left\|u_{n_{k}}\right\|^{2}+b\left\|\nabla u_{n_{k}}\right\|_{2}^{4}+o(1) \\
& \geq b \delta^{4}+o(1) .
\end{aligned}
$$

This contradiction shows that (5.13) holds. Moreover, from (F1) and (3.22), there exist $C_{2}, C_{3}>0$ such that

$$
F(\tau) \geq C_{2}|\tau|^{p}+C_{3}|\tau|^{2}, \quad \forall \tau \in \mathbb{R} .
$$

Then, by (1.7), (5.13), (5.15), the boundedness of $\left\{u_{n}\right\}$, and Sobolev embedding theorem,

$$
\begin{aligned}
I_{\hat{V}}\left(t^{1 / 2}\left(u_{n}\right)_{t}\right) \leq & \frac{a}{2} t^{2}\|\nabla u\|_{2}^{2}+\frac{\hat{V}}{2} t^{4}\left\|u_{n}\right\|_{2}^{2}+\frac{b}{4} t^{4}\left\|\nabla u_{n}\right\|_{2}^{4} \\
& -C_{2} t^{(6+p) / 2}\left\|u_{n}\right\|_{p}^{p}+C_{3} t^{4}\left\|u_{n}\right\|_{2}^{2}-\frac{1}{6} t^{6}\left\|u_{n}\right\|_{6}^{6} \\
& \leq C_{4}\left(t^{2}+t^{4}+t^{(6+p) / 2}\right)-C_{5} t^{6},
\end{aligned}
$$

which together with $p>2$, implies that there exists $T_{2}>0$ such that

$$
I_{\hat{V}}\left(t^{1 / 2}\left(u_{n}\right)_{t}\right)<0, \quad \forall t>T_{2}, n \in \mathbb{N}
$$


Since $I_{\hat{V}}\left(t^{1 / 2}\left(u_{n}\right)_{t}\right) \geq m_{\hat{V}}>0$ by (5.3), (5.16) leads to $t_{n} \leq T_{2}$ for all $n \in \mathbb{N}$, which shows (5.15) holds. Thus it follows from (1.1), (1.7), (2.10), (5.9) and (5.12) that

$$
\begin{aligned}
m_{\varepsilon}+o(1)=\mathcal{J}_{\varepsilon}\left(u_{n}\right) & \geq \mathcal{J}_{\mathcal{E}}\left(t_{n}^{1 / 2}\left(u_{n}\right)_{t_{n}}\right) \\
& \geq I_{\hat{V}}\left(t_{n}^{1 / 2}\left(u_{n}\right)_{t_{n}}\right)+\frac{t_{n}^{4}}{2} \int_{\mathbb{R}^{3}}\left[V\left(\varepsilon t_{n} x\right)-\hat{V}\right] u_{n} \mathrm{~d} x \\
& \geq \hat{m}_{\hat{V}}-\frac{\hat{V} T_{2}^{4}}{2} \int_{|x| \leq R_{0} /\left(\varepsilon T_{1}\right)} u_{n}^{2} \mathrm{~d} x \\
& =\hat{m}_{\hat{V}}+o(1)
\end{aligned}
$$

which, together with Lemma 5.1 and 5.2, implies

$$
m_{\varepsilon} \geq \hat{m}_{\hat{V}} \geq m_{0}+\delta_{0} \geq m_{\varepsilon}+\frac{\delta_{0}}{4} .
$$

This contradiction shows that $\bar{u} \neq 0$. Let $w_{n}=u_{n}-\bar{u}$, by Brezis-Lieb Lemma, one has

$$
\begin{aligned}
& \mathcal{J}_{\varepsilon}\left(u_{n}\right)=\mathcal{J}_{\varepsilon}(\bar{u})+\mathcal{J}_{\varepsilon}\left(w_{n}\right)+\frac{b}{2}\|\nabla \bar{u}\|_{2}^{2}\left\|\nabla w_{n}\right\|_{2}^{2}+o(1) \\
& \mathcal{J}_{\varepsilon}\left(u_{n}\right)=\mathcal{J}_{\varepsilon}(\bar{u})+\mathcal{J}_{\varepsilon}\left(w_{n}\right)+b\|\nabla \bar{u}\|_{2}^{2}\left\|\nabla w_{n}\right\|_{2}^{2}+o(1) .
\end{aligned}
$$

Let

$$
\begin{aligned}
\Psi_{\varepsilon}(u):= & \mathcal{J}_{\varepsilon}(u)-\frac{2}{6+p} \mathfrak{J}_{\varepsilon}(u) \\
= & \frac{(p+2) a}{2(6+p)}\|\nabla u\|_{2}^{2}+\frac{1}{2(6+p)} \int_{\mathbb{R}^{3}}[(p-2) V(\varepsilon x)-2 \nabla V(\varepsilon x) \cdot(\varepsilon x)] u^{2} \mathrm{~d} x \\
& +\frac{(p-2) b}{4(6+p)}\|\nabla u\|_{2}^{4}+\frac{1}{6} \int_{\mathbb{R}^{3}}[f(u) u-p F(u)] \mathrm{d} x+\frac{6-p}{p(6+p)} \int_{\mathbb{R}^{3}} u^{6} \mathrm{~d} x .
\end{aligned}
$$

Then (3.22) and (5.11) imply that $\Psi_{\varepsilon}(u) \geq 0$ for all $u \in H^{1}\left(\mathbb{R}^{3}\right)$. Moreover, it follows from (5.17), (5.18) and (5.19) that

$$
\Psi_{\varepsilon}\left(w_{n}\right) \leq m_{\varepsilon}-\Psi_{\varepsilon}(\bar{u})+o(1), \quad \partial_{\varepsilon}\left(w_{n}\right) \leq-\mathcal{J}_{\varepsilon}(\bar{u})+o(1) .
$$

If there exists a subsequence $\left\{w_{n_{i}}\right\}$ of $\left\{w_{n}\right\}$ such that $w_{n_{i}} \rightarrow 0$, then

$$
\mathcal{J}_{\varepsilon}(\bar{u})=m_{\varepsilon}, \quad J_{\varepsilon}(\bar{u})=0 .
$$

If not, we claim that $\mathcal{J}_{\varepsilon}(\bar{u}) \leq 0$. Otherwise, if $\mathcal{J}_{\varepsilon}(\bar{u})>0$, then (5.20) implies $\mathcal{J}_{\varepsilon}\left(w_{n}\right)<0$ for large $n$. In view of Lemma 2.3, there exists $t_{\varepsilon, n}>0$ such that $\left(t_{\varepsilon, n}\right)^{1 / 2}\left(w_{n}\right)_{t_{\varepsilon, n}} \in \mathcal{M}_{\varepsilon}$. From (1.1), (1.4), (2.3), (5.19) and (5.20), we obtain

$$
\begin{aligned}
m_{\varepsilon}-\Psi(\bar{u})+o(1) \geq \Psi_{\varepsilon}\left(\bar{w}_{n}\right) & =\mathcal{J}_{\varepsilon}\left(w_{n}\right)-\frac{2}{6+p} J_{\varepsilon}\left(w_{n}\right) \\
& \geq \mathcal{J}_{\varepsilon}\left(\left(t_{\varepsilon, n}\right)^{1 / 2}\left(w_{n}\right)_{\varepsilon_{\varepsilon, n}}\right)-\frac{2\left(t_{\varepsilon, n}\right)^{1 / 2}}{6+p} J_{\varepsilon}\left(w_{n}\right) \\
& \geq m_{\varepsilon}-\frac{2\left(t_{\varepsilon, n}\right)^{1 / 2}}{6+p} J_{\varepsilon}\left(w_{n}\right) \geq m_{\varepsilon},
\end{aligned}
$$

which implies $J_{\varepsilon}(\bar{u}) \leq 0$ due to $\Psi_{\varepsilon}(\bar{u})>0$. In view of Lemma 2.3, there exists $t_{\varepsilon}>0$ such that $t_{\varepsilon}^{1 / 2}(\bar{u})_{t_{\varepsilon}} \in \mathcal{M}_{\varepsilon}$. From (1.1), (1.4), (2.3), (3.22), (5.11), (5.19), the weak semi continuity of norm and Fatou's Lemma, one has

$$
\begin{aligned}
m_{\varepsilon} & =\lim _{n \rightarrow \infty}\left[\mathcal{J}_{\mathcal{E}}\left(u_{n}\right)-\frac{2}{6+p} \mathcal{J}_{\varepsilon}\left(u_{n}\right)\right]=\lim _{n \rightarrow \infty} \Psi_{\varepsilon}\left(u_{n}\right) \geq \Psi_{\varepsilon}(\bar{u}) \\
& =\mathcal{J}_{\varepsilon}(\bar{u})-\frac{2}{6+p} \mathcal{J}_{\varepsilon}(\bar{u}) \\
& \geq \mathcal{J}_{\varepsilon}\left(t_{\varepsilon}^{1 / 2}(\bar{u})_{t_{\varepsilon}}\right)-\frac{2 t_{\varepsilon}^{(6+p) / 2}}{6+p} \mathcal{J}_{\varepsilon}(\bar{u}) \geq m_{\varepsilon},
\end{aligned}
$$


which implies (5.21) holds.

In view of Lemma 2.6, 2.7 and 5.3, we can obtain the following Lemma.

Lemma 5.4. Assume that (V1), (V3) and (F1)-(F3) hold. Then for every $\varepsilon \in\left(0, \varepsilon_{0}\right]$, problem $\left(P K_{\varepsilon}\right)$ has a ground state solution $\bar{u}_{\varepsilon}$ such that

$$
\mathcal{J}_{\mathcal{E}}\left(\bar{u}_{\varepsilon}\right)=m_{\varepsilon}=\inf _{u \in H^{1}\left(\mathbb{R}^{3}\right) \backslash\{0\}} \max _{t>0} \mathcal{J}_{\mathcal{E}}\left(t^{1 / 2}(u)_{t}\right)>0 .
$$

\section{Concentration of ground state solutions of $\left(S K_{\varepsilon}\right)$}

In this section, we consider the concentration of ground state solutions of $\left(S K_{\varepsilon}\right)$ and give the proof of Theorem 1.2. For this purpose, we always assume that (V1), (V3) and (F1)-(F3) hold. For every $\varepsilon \in\left[0, \varepsilon_{0}\right]$, let $\bar{u}_{\varepsilon}$ be the ground state solution of $\left(S K_{\varepsilon}\right)$ obtained in Lemma 5.4. Define

$$
\mathcal{L}_{m_{\varepsilon}}=\left\{u \in H^{1}\left(\mathbb{R}^{3}\right) \backslash\{0\}: \mathcal{J}_{\varepsilon}^{\prime}(u)=0, \mathcal{J}_{\varepsilon}(u)=m_{\varepsilon}\right\} \text { and } \Lambda=\left\{u \in \mathcal{L}_{m_{\varepsilon}}: \varepsilon \in\left[0, \varepsilon_{0}\right]\right\} .
$$

Lemma 6.1. There exists a constant $K_{0}>0$, independent of $\varepsilon$, such that $\rho_{0} \leq m_{\varepsilon} \leq K_{0}$ for all $\varepsilon \in\left[0, \varepsilon_{0}\right]$.

Proof. From (2.3), (5.6), Lemmas 2.4 and 2.6, we derive that

$$
\begin{aligned}
\rho_{0} \leq m_{\varepsilon} \leq & \max \left\{\mathcal{J}_{\varepsilon}\left(t^{1 / 2}\left(\hat{u}_{0}\right)_{0}\right): t \in\left(0, T_{0}\right]\right\} \\
\leq & \frac{a T_{0}^{2}}{2}\left\|\nabla \hat{u}_{0}\right\|_{2}^{2}+\frac{V_{\max } T_{0}^{4}}{2}\left\|\hat{u}_{0}\right\|_{2}^{2}+\frac{b T_{0}^{4}}{4}\left\|\nabla \hat{u}_{0}\right\|_{2}^{4} \\
& +C_{1} T_{0}^{4}\left(\left\|\hat{u}_{0}\right\|_{2}^{2}+T_{0}^{4}\left\|\hat{u}_{0}\right\|_{6}^{6}\right):=K_{0}, \quad \forall \varepsilon \in\left[0, \varepsilon_{0}\right] .
\end{aligned}
$$

Lemma 6.2. There exists a constant $K_{1}>0$ independent of $\varepsilon$ such that $\|u\| \leq K_{1}$ for all $u \in \Lambda$.

Proof. Fix $\varepsilon \in\left[0, \varepsilon_{0}\right]$ and $u_{\varepsilon} \in \mathcal{L}_{m_{\varepsilon}}$. Then (2.9) and lemma 6.1 yield

$$
K_{0} \geq m_{\varepsilon}=\mathcal{J}_{\varepsilon}\left(u_{\varepsilon}\right)-\frac{2}{6+p} \mathcal{J}_{\varepsilon}\left(u_{\varepsilon}\right) \geq \frac{b(p-2)}{4(6+p)}\left\|\nabla u_{\varepsilon}\right\|_{2}^{4} .
$$

From (6.1) and (2.14) we deduced that

$$
\left\|u_{\varepsilon}\right\| \leq\left(\frac{2 C_{0}}{\gamma_{1} S^{3}}\right)^{1 / 2}\left\|\nabla u_{\varepsilon}\right\|_{2}^{3} \leq\left(\frac{2 C_{0}}{\gamma_{1} S^{3}}\right)^{1 / 2}\left[\frac{4(6+p) K_{0}}{b(p-2)}\right]^{3 / 4}:=K_{1} .
$$

Lemma 6.3. $\lim \sup _{\varepsilon \rightarrow \bar{\varepsilon}} m_{\varepsilon} \leq m_{\bar{\varepsilon}}$ for every $\bar{\varepsilon} \in\left[0, \varepsilon_{0}\right]$.

Proof. Fix $\bar{\varepsilon} \in\left[0, \varepsilon_{0}\right]$. Arguing by contradiction, suppose that $\lim \sup _{\varepsilon \rightarrow \bar{\varepsilon}} m_{\varepsilon}>m_{\bar{\varepsilon}}$. Let $\epsilon_{0}=\lim \sup _{\varepsilon \rightarrow \bar{\varepsilon}} m_{\varepsilon}-$ $m_{\bar{\varepsilon}}$, then $\epsilon_{0}>0$. From Lemma 2.3, for any $\varepsilon>0$, there exists $\bar{t}_{\varepsilon}>0$ such that $\bar{t}_{\varepsilon}^{1 / 2}\left(\bar{u}_{\bar{\varepsilon}}\right)_{\bar{t}_{\varepsilon}} \in \mathcal{M}_{\varepsilon}$, it follows that

$$
\mathcal{J}_{\varepsilon}\left(\bar{t}_{\varepsilon}^{1 / 2}\left(\bar{u}_{\bar{\varepsilon}}\right)_{\bar{t}_{\varepsilon}}\right) \geq m_{\varepsilon}, \quad \mathcal{J}_{\varepsilon}\left(\bar{t}_{\varepsilon}^{1 / 2}\left(\bar{u}_{\bar{\varepsilon}}\right)_{\bar{t}_{\varepsilon}}\right) \geq \mathcal{J}_{\varepsilon}\left(t^{1 / 2}\left(\bar{u}_{\bar{\varepsilon}}\right)_{t}\right), \quad \forall \varepsilon>0 .
$$

Similar to the proof of (5.6), we easily deduce that there exists a number $\bar{T}>0$ such that $0<\bar{t}_{\varepsilon} \leq \bar{T}$ for any $\varepsilon>0$. It is easy to check that for any bounded set $\Omega \subset \mathbb{R}^{3}$,

$$
\lim _{\varepsilon \rightarrow \bar{\varepsilon}} \sup _{x \in \Omega}[|V(\varepsilon x)-V(\bar{\varepsilon} x)|+|\nabla V(\varepsilon x) \cdot(\varepsilon x)-\nabla V(\bar{\varepsilon} x) \cdot(\bar{\varepsilon} x)|]=0 .
$$

Choose $R_{1}>R_{0}$ such that

$$
V_{\max }\left(2+\bar{T}^{(6+p) / 2}\right) \int_{|x|>R_{1}} \bar{u}_{\bar{\varepsilon}}^{2} \mathrm{~d} x \leq \epsilon_{0} / 2 .
$$


Then it follows from (2.3), (5.11), (6.2)-(6.4) that

$$
\begin{aligned}
& m_{\bar{\varepsilon}}=\mathcal{J}_{\bar{\varepsilon}}\left(\bar{u}_{\bar{\varepsilon}}\right)=\mathcal{J}_{\mathcal{\varepsilon}}\left(\bar{u}_{\bar{\varepsilon}}\right)+\frac{1}{2} \int_{\mathbb{R}^{3}}[V(\bar{\varepsilon} x)-V(\varepsilon x)] \bar{u}_{\bar{\varepsilon}}^{2} \mathrm{~d} x \\
& \geq \mathcal{J}_{\varepsilon}\left(\bar{t}_{\varepsilon}^{1 / 2}\left(\bar{u}_{\bar{\varepsilon}}\right)_{\bar{t}_{\varepsilon}}\right)+\frac{2\left(1-\bar{t}_{\varepsilon}^{(6+p) / 2}\right)}{6+p} \partial_{\varepsilon}\left(\bar{u}_{\bar{\varepsilon}}\right)+\frac{1}{2} \int_{\mathbb{R}^{3}}[V(\bar{\varepsilon} x)-V(\varepsilon x)] \bar{u}_{\bar{\varepsilon}}^{2} \mathrm{~d} x \\
& \geq m_{\varepsilon}+\frac{1-\bar{t}_{\varepsilon}^{(6+p) / 2}}{6+p} \int_{\mathbb{R}^{3}}[4 V(\varepsilon X)+\nabla V(\varepsilon X) \cdot(\varepsilon X)-4 V(\bar{\varepsilon} X)-\nabla V(\bar{\varepsilon} X) \cdot(\bar{\varepsilon} X)] \bar{u}_{\bar{\varepsilon}}^{2} \mathrm{~d} x \\
& +\frac{1}{2} \int_{\mathbb{R}^{3}}[V(\bar{\varepsilon} x)-V(\varepsilon x)] \bar{u}_{\bar{\varepsilon}}^{2} \mathrm{~d} x \\
& \geq m_{\varepsilon}-\frac{1-\bar{t}_{\varepsilon}^{(6+p) / 2}}{6+p} \int_{|x| \leq R_{1}}[4|V(\varepsilon x)-4 V(\bar{\varepsilon} x)|+|\nabla V(\varepsilon x) \cdot(\varepsilon x)-\nabla V(\bar{\varepsilon} x) \cdot(\bar{\varepsilon} x)|] \bar{u}_{\bar{\varepsilon}}^{2} \mathrm{~d} x \\
& -\frac{1}{2} \int_{|x| \leq R_{1}}|V(\bar{\varepsilon} x)-V(\varepsilon x)| \bar{u}_{\bar{\varepsilon}}^{2} \mathrm{~d} x-\left(2+\bar{T}^{(6+p) / 2}\right) V_{\max } \int_{|x| \geq R_{1}} \bar{u}_{\bar{\varepsilon}}^{2} \mathrm{~d} x \\
& \geq m_{\varepsilon}-\frac{1-\bar{T}^{(6+p) / 2}}{6+p}\left\|\bar{u}_{\bar{\varepsilon}}\right\|_{2}^{2} \sup _{|x| \leq R_{1}}[4|V(\varepsilon X)-4 V(\bar{\varepsilon} X)|+|\nabla V(\varepsilon \chi) \cdot(\varepsilon X)-\nabla V(\bar{\varepsilon} X) \cdot(\bar{\varepsilon} X)|] \\
& -\frac{\epsilon_{0}}{2},
\end{aligned}
$$

which implies

$$
m_{\bar{\varepsilon}}+\frac{\epsilon_{0}}{2} \geq m_{\varepsilon} \geq \limsup _{\varepsilon \rightarrow \bar{\varepsilon}} m_{\varepsilon}=m_{\bar{\varepsilon}}+\epsilon_{0}
$$

This contradiction shows that $\lim _{\sup } \rightarrow \bar{\varepsilon} m_{\varepsilon} \leq m_{\bar{\varepsilon}}$.

Lemma 6.4. If $u \in \Lambda$, then $u \in \mathcal{C}\left(\mathbb{R}^{3}, \mathbb{R}\right)$ and $\lim _{|x| \rightarrow \infty} u(x)=0$. Moreover, there is $\alpha_{0}>0$ independent of $x \in \mathbb{R}^{3}$ and $u \in \Lambda$ such that

$$
|u(x)| \leq \alpha_{0} \int_{B_{1}(x)}|u(y)| \mathrm{d} y, \quad \forall x \in \mathbb{R}^{3}, u \in \Lambda .
$$

Proof. For $s \geq 2$, it follows from Lemma 6.2 and the standard bootstrap argument (see [6, 29]) that there exists $C_{s}>0$ independent of $u \in \Lambda$ such that

$$
u \in W^{1, s}\left(\mathbb{R}^{3}\right), \quad\|u\|_{W^{1, s}\left(\mathbb{R}^{3}\right)} \leq C_{s}, \quad \forall u \in \Lambda,
$$

which, together with Sobolev imbedding theorem, implies that there is $C_{\infty}>0$ independent of $u \in \Lambda$ such that

$$
\|u\|_{\infty} \leq C_{\infty}, \quad \forall u \in \Lambda .
$$

By (F1), there exists a constant $\Theta_{1}>V_{0}$ such that

$$
\left|f(t)+t^{5}\right| \leq \Theta_{1}|t|, \quad \forall|t| \leq C_{\infty} .
$$

From (6.6), (6.7), Lemma 6.2 and [29, Lemma 1], we have $u \in \mathcal{C}\left(\mathbb{R}^{3}, \mathbb{R}\right)$ and $\lim _{|x| \rightarrow \infty} u(x)=0$. Since $u \in \mathcal{L}_{m_{\varepsilon}}$ is a solution of $\left(P K_{\varepsilon}\right)$ for some $\varepsilon>0$, then it follows from (5.7) and Lemma 6.2 that

$$
\begin{aligned}
\nabla u & =\frac{u \cdot \nabla u}{|u|}=\frac{V(\varepsilon x) u^{2}-f(u) u-u^{6}}{\left(a+b\|\nabla u\|_{2}^{2}\right)|u|} \\
& \geq \frac{V_{0}-\Theta_{1}}{a+b K_{1}^{2}}|u|:=-\varrho_{0}|u|, \quad \forall x \in \mathbb{R}^{3},
\end{aligned}
$$

which implies that $|u|$ is a sub-solution of the equation $\left(-\Delta-\varrho_{0}\right) w=0$, and hence (6.5) follows from the sub-solution estimate (see [33, Theorem C. 12]). 
Lemma 6.5. For every $u_{\varepsilon} \in \mathcal{L}_{m_{\varepsilon}} \subset \Lambda$, there exists $y_{\varepsilon} \in \mathbb{R}^{3}$ such that $\left|u_{\varepsilon}\left(y_{\varepsilon}\right)\right|=\max _{x \in \mathbb{R}^{3}}\left|u_{\varepsilon}(x)\right|$. Let $\tilde{u}_{\varepsilon}(x):=$ $u_{\varepsilon}\left(x+y_{\varepsilon}\right)$ with $u_{\varepsilon} \in \mathcal{L}_{m_{\varepsilon}} \subset \Lambda$, and let $\varepsilon_{n} \in\left(0, \varepsilon_{0}\right]$ such that $\limsup _{n \rightarrow \infty} \varepsilon_{n}=\bar{\varepsilon}$. Then we have

(i) if $\bar{\varepsilon}>0$, then $\left\{u_{\varepsilon_{n}}\right\}$ has a convergence subsequence, whose limit belongs to $\Lambda$;

(ii) if $\bar{\varepsilon}=0$, then $\left\{\tilde{u}_{\varepsilon_{n}}\right\}$ has a convergence subsequence, whose limit is not zero.

Proof. For $\left\{\varepsilon_{n}\right\} \subset\left[0, \varepsilon_{0}\right]$ and $u_{\varepsilon_{n}} \subset \mathcal{L}_{m_{\varepsilon_{n}}}$, Lemma 6.2 implies that $\left\{u_{\varepsilon_{n}}\right\}$ is bounded in $H^{1}\left(\mathbb{R}^{3}\right)$. Since $0<m_{\varepsilon}<$ $m_{1}^{\infty}<\frac{a b S^{3}}{4}+\frac{b^{3} S^{6}}{24}+\frac{\left(b^{2} S^{4}+4 a S\right)^{3 / 2}}{24}$ due to Lemma 4.4, as in the proof of (3.25), we have

$$
\limsup _{n \rightarrow \infty} \sup _{y \in \mathbb{R}^{3}} \int_{B_{1}(y)}\left|u_{\varepsilon_{n}}\right|^{2} \mathrm{~d} x>0 .
$$

By Lemma 6.4, there exists $y_{\varepsilon} \in \mathbb{R}^{3}$ such that $\left|u_{\varepsilon}\left(y_{\varepsilon}\right)\right|=\max _{x \in \mathbb{R}^{3}}\left|u_{\varepsilon}(x)\right|$. Then it follows from (6.9) that

$$
\limsup _{n \rightarrow \infty}\left|u_{\varepsilon_{n}}\left(y_{\varepsilon_{n}}\right)\right|^{2} \geq \frac{3}{4 \pi} \limsup _{n \rightarrow \infty} \sup _{y \in \mathbb{R}^{3}} \int_{B_{1}(y)}\left|u_{\varepsilon_{n}}\right|^{2} \mathrm{~d} x>0 .
$$

By (1.1), (1.4) and (5.19), one has

$$
m_{\varepsilon_{n}}=\mathcal{J}_{\varepsilon_{n}}\left(u_{\varepsilon_{n}}\right)-\frac{2}{6+p} \mathcal{J}_{\varepsilon_{n}}\left(u_{\varepsilon_{n}}\right)=\Psi_{\varepsilon_{n}}\left(u_{\varepsilon_{n}}\right)>0 .
$$

In the following we prove that (i) or (ii) hold.

(i) If $\bar{\varepsilon} \in\left(0, \varepsilon_{0}\right]$, then passing to a subsequence, we may assume that

$$
\varepsilon_{n} \rightarrow \bar{\varepsilon} \in\left(0, \varepsilon_{0}\right], \quad u_{\varepsilon_{n}} \rightarrow \bar{u} \text { in } H^{1}\left(\mathbb{R}^{3}\right) .
$$

By Lemma 5.1 and 5.2, $m_{\varepsilon_{n}} \leq \hat{m}_{\hat{V}}-\delta_{0} / 4$. Since $u_{\varepsilon_{n}} \neq 0$, then it follows from Lemma 2.3 that there exists $\hat{t}_{n}>0$ such that $\hat{t}_{n}^{1 / 2}\left(u_{\varepsilon_{n}}\right)_{\hat{t}_{n}} \in \hat{\mathcal{M}}_{\hat{V}}$, and so $I_{\hat{V}}\left(\hat{t}_{n}^{1 / 2}\left(u_{\varepsilon_{n}}\right)_{\hat{t}_{n}}\right) \geq \hat{M}_{\hat{V}}$. Similar to the proof of (5.12), there exist $\hat{T}_{1}, \hat{T}_{2}>0$ such that $0<\hat{T}_{1} \leq \hat{t}_{n} \leq \hat{T}_{2}$. If $\bar{u}=0$, then $u_{\varepsilon_{n}} \rightarrow 0$ in $H^{1}\left(\mathbb{R}^{3}\right), u_{\varepsilon_{n}} \rightarrow 0$ in $L_{l o c}^{s}\left(\mathbb{R}^{3}\right), 2 \leq s<2^{\star}$ and $u_{\varepsilon_{n}} \rightarrow 0$ a.e. on $\mathbb{R}^{3}$. Then it follows from (1.1), (1.7), (5.1), (5.9) and (6.12) that

$$
\begin{aligned}
\hat{m}_{\hat{V}}-\delta_{0} / 4 & \geq m_{\varepsilon_{n}}=\mathcal{J}_{\varepsilon_{n}}\left(u_{\varepsilon_{n}}\right) \geq \mathcal{J}_{\varepsilon_{n}}\left(\hat{t}_{n}^{1 / 2}\left(u_{\varepsilon_{n}}\right)_{\hat{t}_{n}}\right) \\
& =I_{\hat{V}}\left(\hat{t}_{n}^{1 / 2}\left(u_{\varepsilon_{n}}\right)_{\hat{t}_{n}}\right)+\frac{\hat{t}_{n}^{4}}{2} \int_{\mathbb{R}^{3}}\left[V\left(\varepsilon_{n} \hat{t}_{n} x\right)-\hat{V}\right]\left|u_{\varepsilon_{n}}\right|^{2} \mid \mathrm{d} x \\
& \geq \hat{m}_{\hat{V}}-\frac{2 V_{\max }-V_{\infty}-V(0)}{4} \hat{T}_{2}^{4} \int_{|x| \leq R_{0} /\left(\hat{T}_{1} \varepsilon_{n}\right)}\left|u_{\varepsilon_{n}}\right|^{2} \mathrm{~d} x \\
& =\hat{m}_{\hat{V}}+o(1) .
\end{aligned}
$$

This contradiction shows that $\bar{u} \neq 0$.

Let $w_{\varepsilon_{n}}=u_{\varepsilon_{n}}-\bar{u}$. Then by Brezis-Lieb Lemma, one has

$$
\mathcal{J}_{\mathcal{E}_{n}}\left(u_{\varepsilon_{n}}\right)=\mathcal{J}_{\bar{\varepsilon}}(\bar{u})+\mathcal{J}_{\mathcal{E}_{n}}\left(w_{\varepsilon_{n}}\right)+\frac{b}{2}\|\nabla \bar{u}\|_{2}^{2}\left\|\nabla w_{\varepsilon}\right\|_{2}^{2}+o(1),
$$

and

$$
\partial_{\varepsilon_{n}}\left(u_{\varepsilon_{n}}\right)=\mathcal{J}_{\bar{\varepsilon}}(\bar{u})+\partial_{\varepsilon_{n}}\left(w_{\varepsilon_{n}}\right)+b\|\nabla \bar{u}\|_{2}^{2}\left\|\nabla w_{\varepsilon}\right\|_{2}^{2}+o(1) .
$$

Moreover, it follows from (5.19), (6.12), (6.14) and (6.15) that

$$
\Psi_{\varepsilon_{n}}\left(u_{\varepsilon_{n}}\right) \leq m_{\varepsilon_{n}}-\Psi_{\bar{\varepsilon}}(\bar{u})+o(1), \quad \partial_{\varepsilon_{n}}\left(w_{\varepsilon_{n}}\right) \leq-\partial_{\varepsilon_{n}}(\bar{u})+o(1) .
$$

Now we prove that

$$
u_{\varepsilon_{n}} \rightarrow \bar{u} \text { in } H^{1}\left(\mathbb{R}^{3}\right), \quad \mathcal{J}_{\bar{\varepsilon}}(\bar{u})=m_{\bar{\varepsilon}}, \quad J_{\bar{\varepsilon}}(\bar{u})=0 .
$$


If there exists a subsequence $\left\{w_{\varepsilon_{n_{i}}}\right\}$ of $\left\{w_{\varepsilon_{n}}\right\}$ such that $\lim _{n_{i} \rightarrow \infty} w_{\varepsilon_{n_{i}}}=0$, then (6.17) holds. If not, we claim that $\mathcal{J}_{\bar{\varepsilon}}(\bar{u}) \leq 0$. Otherwise, if $\mathcal{J}_{\bar{\varepsilon}}(\bar{u})>0$, then (6.15) implies that $\mathcal{J}_{\varepsilon_{n}}\left(w_{\varepsilon_{n}}\right)<0$ for large $n$. In view of Lemma 2.3, there exists $t_{n}>0$ such that $t_{n}^{1 / 2}\left(w_{\varepsilon_{n}}\right)_{t_{n}} \in \mathcal{M}_{\bar{\varepsilon}}$. From (1.1), (1.4), (2.3), (5.19)and (6.15), we obtain

$$
\begin{aligned}
m_{\varepsilon_{n}}-\Psi_{\bar{\varepsilon}}(\bar{u})+o(1) & =\Psi_{\varepsilon_{n}}\left(w_{\varepsilon_{n}}\right)=\mathcal{J}_{\varepsilon_{n}}\left(w_{\varepsilon_{n}}\right)-\frac{2}{6+p} \mathcal{J}_{\varepsilon_{n}}\left(w_{\varepsilon_{n}}\right) \\
& \geq \mathcal{J}\left(t_{n}^{1 / 2}\left(w_{\varepsilon_{n}}\right) t_{n}\right)-\frac{2 t_{n}^{(6+p) / 2}}{6+p} \mathcal{J}_{\varepsilon_{n}}\left(w_{\varepsilon_{n}}\right) \\
& \geq m_{\varepsilon_{n}}-\frac{2 t_{n}^{(6+p) / 2}}{6+p} \partial_{\varepsilon_{n}}\left(w_{\varepsilon_{n}}\right) \geq m_{\varepsilon_{n}},
\end{aligned}
$$

which is a contradiction due to $\Psi_{\bar{\varepsilon}}(\bar{u})>0$. Hence, $\partial_{\bar{\varepsilon}}(\bar{u}) \leq 0$. In view of Lemma 2.3, there exists $\bar{t}>0$ such that $\bar{t}^{1 / 2}(\bar{u})_{\bar{t}} \in \mathcal{M}_{\bar{\varepsilon}}$. Then it follows from (2.3), (5.11), (5.19), (6.11), the weak semicontinuity of norm, Fatou's Lemma and Lemma 6.3 that

$$
\begin{aligned}
m_{\bar{\varepsilon}} & \geq \limsup _{n \rightarrow \infty} m_{\varepsilon_{n}}=\limsup _{n \rightarrow \infty} \Psi_{\varepsilon_{n}}\left(u_{\varepsilon_{n}}\right) \geq \liminf _{n \rightarrow \infty} \Psi_{\varepsilon_{n}}\left(u_{\varepsilon_{n}}\right) \geq \Psi_{\bar{\varepsilon}}(\bar{u}) \\
& =\mathcal{J}_{\bar{\varepsilon}}(\bar{u})-\frac{2}{6+p} \mathcal{J}_{\bar{\varepsilon}}(\bar{u}) \geq \mathcal{J}_{\bar{\varepsilon}}\left(\bar{t}^{1 / 2}(\bar{u})_{\bar{t}}\right)-\frac{2 \bar{t}^{(6+p) / 2}}{6+p} \mathcal{J}_{\bar{\varepsilon}}(\bar{u}) \\
& \geq m_{\bar{\varepsilon}}-\frac{2 \bar{t}^{(6+p) / 2}}{6+p} \mathcal{J}_{\bar{\varepsilon}}(\bar{u}) \geq m_{\bar{\varepsilon}}
\end{aligned}
$$

which implies that $\mathcal{\partial}_{\bar{\varepsilon}}=0$,

$$
\mathcal{J}_{\varepsilon_{n}}\left(u_{\varepsilon_{n}}\right)=m_{\varepsilon_{n}} \rightarrow m_{\bar{\varepsilon}}=\mathcal{J}_{\bar{\varepsilon}}(\bar{u})
$$

and

$$
\Psi_{\varepsilon_{n}}\left(u_{\varepsilon_{n}}\right) \rightarrow \Psi_{\bar{\varepsilon}}(\bar{u}) .
$$

Next, we show that $u_{\varepsilon_{n}} \rightarrow \bar{u}$ in $H^{1}\left(\mathbb{R}^{3}\right)$. From (5.19), (6.12) and (6.19), we can deduce that $u_{\varepsilon_{n}} \rightarrow \bar{u}$ in $\mathcal{D}^{1,2}\left(\mathbb{R}^{3}\right)$, and so $u_{\varepsilon_{n}} \rightarrow \bar{u}$ in $L^{s}\left(\mathbb{R}^{3}\right)$ for $2<s \leq 6$. Jointly with (6.18), one has

$$
\int_{\mathbb{R}^{3}} V\left(\varepsilon_{n} x\right) u_{\varepsilon_{n}}^{2} \mathrm{~d} x \rightarrow \int_{\mathbb{R}^{3}} V(\bar{\varepsilon} x) \bar{u}^{2} \mathrm{~d} x .
$$

We claim that $u_{\varepsilon_{n}} \rightarrow \bar{u}$ in $L^{2}\left(\mathbb{R}^{3}\right)$. In fact, for any $\epsilon>0$, there exists $R_{\epsilon}>0$ large enough such that

$$
\int_{|x| \geq R_{\epsilon}} V(\bar{\varepsilon} x) \bar{u}^{2} \mathrm{~d} x \leq \epsilon .
$$

Since $u_{\varepsilon_{n}} \rightarrow \bar{u}$ in $L^{2}\left(B_{R_{\varepsilon}}(0)\right)$, it follows from (6.20) and (6.21) that

$$
\lim _{n \rightarrow \infty} \int_{|x| \geq R_{\epsilon}} V\left(\varepsilon_{n} x\right) u_{\varepsilon_{n}}^{2} \mathrm{~d} x=\int_{|x| \geq R_{\epsilon}} V(\bar{\varepsilon} x) \bar{u}^{2} \mathrm{~d} x \leq \epsilon,
$$

which implies

$$
\begin{aligned}
\lim _{n \rightarrow \infty} \int_{\mathbb{R}^{3}} V_{0}\left|u_{\varepsilon_{n}}-\bar{u}\right|^{2} \mathrm{~d} x & =\lim _{n \rightarrow \infty} \int_{|x| \geq R_{\epsilon}} V_{0}\left|u_{\varepsilon_{n}}-\bar{u}\right|^{2} \mathrm{~d} x \\
& \leq \lim _{n \rightarrow \infty} \int_{|x| \geq R_{\epsilon}} V\left(\varepsilon_{n} x\right) u_{\varepsilon_{n}}^{2} \mathrm{~d} x+\int_{|x| \geq R_{\epsilon}} V(\bar{\varepsilon} x) \bar{u}^{2} \mathrm{~d} x \leq 2 \epsilon .
\end{aligned}
$$

By the arbitrariness of $\epsilon>0$, we derive from (6.23) that $u_{\varepsilon_{n}} \rightarrow \bar{u}$ in $L^{2}\left(\mathbb{R}^{3}\right)$. Hence, $u_{\varepsilon_{n}} \rightarrow \bar{u}$ in $H^{1}\left(\mathbb{R}^{3}\right)$, and so (6.17) holds. Combining with Lemma 2.7 with (6.17), we have $\mathcal{J}_{\bar{u}}^{\prime}(\bar{u})=0$. This shows that $\bar{u} \in \mathcal{L}_{m_{\bar{\varepsilon}}} \subset \Lambda$.

(ii) If $\bar{\varepsilon}=0$, passing to a subsequence, we may assume that

$$
\varepsilon_{n} \rightarrow 0, \quad \tilde{u} \rightarrow \tilde{u}_{0} \text { in } H^{1}\left(\mathbb{R}^{3}\right) .
$$


Moreover, (6.10) implies that $\tilde{u}_{0} \neq 0$. Since $\mathrm{V}$ is bounded, going to a subsequence if necessary, we may assume that

$$
\lim _{n \rightarrow \infty} V\left(\varepsilon_{n} y_{\varepsilon_{n}}\right)=\beta>0
$$

In view of Theorem 1.3, there exists $\hat{u}_{\beta} \in \hat{\mathcal{M}}_{\beta}$ such that

$$
I_{\beta}\left(\hat{u}_{\beta}\right)=\hat{m}_{\beta}, \quad I_{\beta}^{\prime}\left(\hat{u}_{\beta}\right)=0 \text {. }
$$

Note that

$$
\begin{aligned}
m_{\varepsilon_{n}}=\mathcal{J}_{\varepsilon_{n}}\left(u_{\varepsilon_{n}}\right)= & \frac{a}{2}\left\|\nabla \hat{u}_{\varepsilon_{n}}\right\|+\frac{1}{2} \int_{\mathbb{R}^{3}} V\left(\varepsilon_{n}\left(x+y_{\varepsilon_{n}}\right)\right)\left|\hat{u}_{\varepsilon_{n}}\right|^{2} \mathrm{~d} x+\frac{b}{4}\left\|\nabla \hat{u}_{\varepsilon_{n}}\right\|_{2}^{4} \\
& -\int_{\mathbb{R}^{3}}\left[F\left(\hat{u}_{\varepsilon_{n}}\right)+\frac{1}{6}\left|\hat{u}_{\varepsilon_{n}}\right|^{6}\right] \mathrm{d} x .
\end{aligned}
$$

It is easy to check that for any bounded set $\Omega \subset \mathbb{R}^{3}$,

$$
\lim _{n \rightarrow \infty} \sup _{x \in \Omega} V\left(\varepsilon_{n}\left(x+y_{\varepsilon_{n}}\right)\right)=\beta
$$

Arguing as in the proof of Lemma 6.3,

$$
\limsup _{n \rightarrow \infty} m_{\varepsilon_{n}} \leq \hat{m}_{\beta}
$$

Analogous to the proof of (6.17) in Case (i),

$$
\tilde{u}_{\varepsilon_{n}} \rightarrow \tilde{u}_{0} \text { in } H^{1}\left(\mathbb{R}^{3}\right), I_{\beta}\left(\tilde{u}_{0}\right)=\hat{m}_{\beta}=\limsup _{n \rightarrow \infty} m_{\varepsilon_{n}}, J_{\beta}\left(\tilde{u}_{0}\right)=0 .
$$

Lemma 6.6. $\inf \left\{\|u\|_{\infty}: u \in \Lambda\right\}:=\delta_{0}>0$.

Proof. Suppose to the contrary that $\delta_{0}=0$. Then there is a sequence $\left\{u_{\varepsilon_{n}}\right\} \subset \Lambda$ such that $\lim _{n \rightarrow \infty}\|u\|_{\infty}=0$. Let $u_{\varepsilon_{n}} \in \mathcal{L}_{m_{\varepsilon_{n}}}$. There are two possible cases.

Case (i) $\lim \sup _{n \rightarrow \infty} \varepsilon_{n}>0$. By Lemma 6.5(i), there exists $\bar{u} \in \Lambda$ such that $u_{\varepsilon_{n}} \rightarrow \bar{u}$ in $H^{1}\left(\mathbb{R}^{3}\right)$. Then it follows from the Hölder inequality that

$$
\begin{aligned}
\int_{B_{1}(x)}|\bar{u}(y)| \mathrm{d} x & \leq \int_{B_{1}(x)}\left|u_{\varepsilon_{n}}(y)-\bar{u}(y)\right| \mathrm{d} y+\int_{B_{1}(x)}\left|u_{\varepsilon_{n}}(y)\right| \mathrm{d} y \\
& \leq\left(\frac{4 \pi}{3}\right)^{1 / 2}\left\|u_{\varepsilon_{n}}-\bar{u}\right\|_{2}+\frac{4 \pi}{3}\left\|\bar{u}_{n}\right\|_{\infty}=o(1), \quad \forall x \in \mathbb{R}^{3},
\end{aligned}
$$

which implies that $\bar{u}=0$, a contradiction.

Case (ii) $\lim \sup _{n \rightarrow \infty} \varepsilon_{n}=0$. By Lemma 6.5(ii), there exists $\tilde{u}_{0} \in H^{1}\left(\mathbb{R}^{3}\right) \backslash\{0\}$ such that $\tilde{u}_{n} \rightarrow \tilde{u}_{0}$ in $H^{1}\left(\mathbb{R}^{3}\right)$. Then it follows from the Hölder inequality that

$$
\begin{aligned}
\int_{B_{1}(x)}\left|\tilde{u}_{0}(y)\right| \mathrm{d} x & \leq \int_{B_{1}(x)}\left|\tilde{u}_{n}(y)-\tilde{u}_{0}(y)\right| \mathrm{d} y+\int_{B_{1}(x)}\left|\tilde{u}_{n}(y)\right| \mathrm{d} y \\
& \leq\left(\frac{4 \pi}{3}\right)^{1 / 2}\left\|\tilde{u}_{n}-\tilde{u}_{0}\right\|_{2}+\frac{4 \pi}{3}\left\|\tilde{u}_{n}\right\|_{\infty}=o(1), \quad \forall x \in \mathbb{R}^{3},
\end{aligned}
$$

which implies that $\tilde{u}_{0}=0$, a contradiction.

Lemma 6.7. There exist $\Pi_{1}, \kappa_{1}>0$ independent of $x \in \mathbb{R}^{3}$ and $u \in \Lambda$ such that

$$
|u(x)| \leq \Pi_{1} \exp \left(-\kappa_{1}\left|x-y_{u}\right|\right), \quad \forall x \in \mathbb{R}^{3}, u \in \Lambda,
$$

where $\left|u\left(y_{u}\right)\right|=\max _{x \in \mathbb{R}^{3}}|u(x)|$. 
Proof. First, we claim that $u(x) \rightarrow 0$, as $\left|x-y_{u}\right| \rightarrow \infty$, uniformly in $u \in \Lambda$. In fact, if not, then there exist $\delta_{1}>0,\left\{u_{n}\right\} \subset \Lambda$ and $\left\{x_{n}\right\} \in \mathbb{R}^{3}$ with $\left|x_{n}-y_{u_{n}}\right| \rightarrow \infty$ such that $\left|u_{n}\left(x_{n}\right)\right| \geq \delta_{1}$. Let $u_{n} \in \mathcal{L}_{m_{\varepsilon_{n}}}$. There are two possible cases.

Case (i) $\lim _{\sup _{n \rightarrow \infty}} \varepsilon_{n}>0$. By Lemma 6.5(i), there exists $u_{\star} \in \Lambda$ such that $u_{n} \rightarrow u_{\star}$ in $H^{1}\left(\mathbb{R}^{3}\right)$. Since $\left|x_{n}-y_{u_{n}}\right| \rightarrow \infty$, then either $\lim \sup _{n \rightarrow \infty}\left|x_{n}\right|=\infty$ or $\limsup _{n \rightarrow \infty}\left|y_{u_{n}}\right|=\infty$, and so we deduce from (6.5) and the Hölder inequality that

$$
\delta_{1} \leq\left|u_{n}\left(x_{n}\right)\right| \leq \alpha_{0} \int_{B_{1}\left(x_{n}\right)}\left|u_{n}(y)\right| \mathrm{d} y=\alpha_{0} \int_{B_{1}\left(x_{n}\right)}\left|u_{*}(y)\right| \mathrm{d} y+o(1)=o(1)
$$

and

$$
\delta_{1} \leq\left|u_{n}\left(y_{u_{n}}\right)\right| \leq \alpha_{0} \int_{B_{1}\left(y_{u_{n}}\right)}\left|u_{n}(y)\right| \mathrm{d} y=\alpha_{0} \int_{B_{1}\left(y_{u_{n}}\right)}\left|u_{*}(y)\right| \mathrm{d} y+o(1)=o(1),
$$

a contradiction.

Case (ii) $\lim \sup _{n \rightarrow \infty} \varepsilon_{n}=0$. By Lemma 6.5(ii), there exists $\tilde{u}_{\star} \in H^{1}\left(\mathbb{R}^{3}\right) \backslash\{0\}$ such that $\tilde{u}_{n} \rightarrow \tilde{u}_{\star}$ in $H^{1}\left(\mathbb{R}^{3}\right)$. Then it follows from (6.5) and the Hölder inequality that

$$
\begin{aligned}
\delta_{1} & \leq\left|u_{n}\left(x_{n}\right)\right| \leq \alpha_{0} \int_{B_{1}\left(x_{n}\right)}\left|u_{n}(y)\right| \mathrm{d} y=\alpha_{0} \int_{B_{1}\left(x_{n}-y_{u_{n}}\right)}\left|\tilde{u}_{n}(y)\right| \mathrm{d} y \\
& \leq \alpha_{0} \int_{B_{1}\left(x_{n}-y_{u_{n}}\right)}\left|\tilde{u}_{\star}(y)\right| \mathrm{d} y+o(1)=o(1),
\end{aligned}
$$

a contradiction.

By (F1) and the above claim, there exists a number $R_{2}>0$ independent of $x \in \mathbb{R}^{3}$ and $u \in \Lambda$, such that for any $u \in \Lambda$

$$
\left|f(u(x))+u^{5}(x)\right| \leq \frac{V_{0}}{2}|u(x)| \text { for all } x, \text { with }\left|x-y_{u}\right| \geq R_{2} .
$$

Substitute (6.33) to (6.8), one has

$$
\begin{aligned}
\Delta|u| & =\frac{u \cdot \Delta u}{|u|}=\frac{V(\varepsilon x) u^{2}-f(u) u-u^{5}}{\left(a+b\|\nabla u\|_{2}^{2}\right)|u|} \\
& \geq \frac{V_{0}}{2\left(a+b K_{1}^{2}\right)}|u|:=\varrho_{1}|u|, \quad \forall x \in \mathbb{R}^{3},\left|x-y_{u}\right| \geq R_{2} .
\end{aligned}
$$

Set $w(x)=|u(x)|-C_{\infty} e^{-\sqrt{\varrho_{1}}\left(\left|x-y_{u}\right|-R_{2}\right)}$, where $C_{\infty}$ is given by (6.6). Then

$$
\Delta w \geq \varrho_{1} w,\left|x-y_{u}\right| \geq R_{2} .
$$

By the maximum principle, we can conclude that $w(x) \leq 0$ for $\left|x-y_{u}\right| \geq R_{2}$ i.e.,

$$
w(x) \leq C_{\infty} e^{-\sqrt{\varrho_{1}}\left(\left|x-y_{u}\right|-R_{2}\right)},\left|x-y_{u}\right| \geq R_{2} .
$$

Therefore, the claim (6.32) holds.

Lemma 6.8. Let $u_{\varepsilon} \in \mathcal{L}_{m_{\varepsilon}}$ for $\varepsilon \in\left(0, \varepsilon_{0}\right]$ and let $y_{\varepsilon} \in \mathbb{R}^{3}$ be a global maximum point of $u_{\varepsilon}$. Then (i) $\sup _{\varepsilon \in\left[0, \varepsilon_{0}\right]}\left(\varepsilon\left|y_{\varepsilon}\right|\right)<\infty$;

(i) for $\varepsilon \rightarrow 0^{+}$, up to a subsequence, $\tilde{u}_{\varepsilon_{n}}=u_{\varepsilon_{n}}\left(\cdot+y_{\varepsilon_{n}}\right)$ converges in $H^{1}\left(\mathbb{R}^{3}\right)$ to a ground state solution of $\left(K_{V_{0}}\right)$.

Proof. (i) Assume by contradiction that there exists a sequence $\left\{\varepsilon_{n}\right\} \subset\left[0, \varepsilon_{0}\right]$ such that $\varepsilon_{n}\left|y_{\varepsilon_{n}}\right| \rightarrow \infty$. There are two possible cases.

Case (1) $\lim \sup _{n \rightarrow \infty} \varepsilon_{n}=\bar{u} \in\left(0, \varepsilon_{0}\right]$. In this case, by Lemma 6.5(i), up to a subsequence, one has $\left|y_{\varepsilon_{n}}\right| \rightarrow \infty$ and $u_{\varepsilon_{n}} \rightarrow u_{\bar{\varepsilon}} \in \Lambda$ in $H^{1}\left(\mathbb{R}^{3}\right)$. Hence, it follows from (6.5) and the Hölder inequality that

$$
\delta_{0} \leq\left|u_{\varepsilon_{n}}\left(y_{\varepsilon_{n}}\right)\right| \leq \alpha_{0} \int_{B_{1}\left(y_{\varepsilon_{n}}\right)}\left|u_{\varepsilon_{n}}(y)\right| \mathrm{d} y=\alpha_{0} \int_{B_{1}\left(y_{\varepsilon_{n}}\right)}\left|u_{\bar{\varepsilon}}(y)\right| \mathrm{d} y+o(1)=o(1),
$$


a contradiction.

Case (2) $\lim \sup _{n \rightarrow \infty} \varepsilon_{n}=0$. In this case, by (6.27), (6.30) and Lemma 6.3 that

$$
\hat{m}_{\beta}=\limsup _{n \rightarrow \infty} m_{\varepsilon_{n}} \leq m_{0} .
$$

In the view of (6.26) and Lemma 2.3, there exists $\tilde{t}>0$ such that $\tilde{t}^{1 / 2}\left(\hat{u}_{\beta}\right)_{\tilde{t}} \in \mathcal{M}_{0}$, and so $\mathcal{J}_{0}\left(\tilde{t}^{1 / 2}\left(\hat{u}_{\beta}\right)_{\tilde{t}}\right) \geq m_{0}$. Note that

$$
V(0)=\min _{x \in \mathbb{R}^{3}} V(x)<V_{\infty} \leq \lim _{n \rightarrow \infty} V\left(\varepsilon_{n}\left(x+y_{\varepsilon_{n}}\right)\right)=\beta, \quad \forall x \in \mathbb{R}^{3} .
$$

Applying Corollary 2.1 to $I_{\beta}$, we derive from (1.7), (6.26), (6.35) and (6.36) that

$$
\begin{aligned}
\hat{m}_{\beta} & =I_{\beta}\left(\hat{u}_{\beta}\right) \geq I_{\beta}\left(\tilde{t}^{1 / 2}\left(\hat{u}_{\beta}\right)_{\tilde{t}}\right) \\
& =\mathcal{J}_{0}\left(\tilde{t}^{1 / 2}\left(\hat{u}_{\beta}\right)_{\tilde{t}}\right)+\frac{\beta-V(0)}{2} \tilde{t}^{4}\left\|\hat{u}_{\beta}\right\|_{2}^{2} \\
& \geq m_{0}+\frac{\beta-V(0)}{2} \tilde{t}^{4}\left\|\hat{u}_{\beta}\right\|_{2}^{2}>m_{0} \geq \hat{m}_{\beta},
\end{aligned}
$$

a contradiction. Case (1) and (2) show that $\sup _{\varepsilon \in\left[0, \varepsilon_{0}\right]}\left(\varepsilon\left|y_{\varepsilon}\right|\right)<\infty$.

(ii) In view of Lemma 6.2, $\left\{u_{\varepsilon_{n}}\right\}$ is bounded in $H^{1}\left(\mathbb{R}^{3}\right)$, and so $\left\{\tilde{u}_{\varepsilon_{n}}\right\}$ is bounded in $H^{1}\left(\mathbb{R}^{3}\right)$. After extracting a subsequence, we may assume that $\tilde{u}_{\varepsilon_{n}} \rightarrow \tilde{u}$ in $H^{1}\left(\mathbb{R}^{3}\right), \tilde{u}_{\varepsilon_{n}} \rightarrow \tilde{u}$ in $L_{l o c}^{s}\left(\mathbb{R}^{3}\right), 2 \leq s<2^{\star}$ and $\tilde{u}_{\varepsilon_{n}} \rightarrow \tilde{u}$ a.e. on $\mathbb{R}^{3}$. It follows from (6.5) and Lemma 6.6 that

$$
\begin{aligned}
\delta_{0} \leq\left|\tilde{u}_{\varepsilon_{n}}(0)\right|=\left|u_{\varepsilon_{n}}\left(y_{\varepsilon_{n}}\right)\right| & \leq \alpha_{0} \int_{B_{1}\left(y_{\varepsilon_{n}}\right)}\left|u_{\varepsilon_{n}}(y)\right| \mathrm{d} y \\
& =\alpha_{0} \int_{B_{1}(0)}\left|\tilde{u}_{\varepsilon_{n}}(y)\right| \mathrm{d} y=\alpha_{0} \int_{B_{1}(0)}|\tilde{u}(y)| \mathrm{d} y+o(1) .
\end{aligned}
$$

This shows $\tilde{u} \neq 0$. Moreover, by item (i), there exists $\tilde{y} \in \mathbb{R}^{3}$ such that, up to a subsequence, $\varepsilon_{n} y_{\varepsilon_{n}} \rightarrow \tilde{y}$.

Next, we prove that $\tilde{u}_{\varepsilon_{n}} \rightarrow \tilde{u}$ in $H^{1}\left(\mathbb{R}^{3}\right), V(\tilde{y})=V(0)$ and $\tilde{u}$ is the ground state solution of $\left(K_{V_{0}}\right)$. Let $\hat{V}:=V(\tilde{y})$. Since $V_{0} \leq \tilde{V}$, then it follows from Lemma 6.3 that

$$
\limsup _{n \rightarrow \infty} m_{\varepsilon_{n}} \leq m_{0} \leq \hat{m}_{\tilde{V}} .
$$

Analogous to the proof of (6.30), we deduce that as $n \rightarrow \infty$

$$
I_{\tilde{V}}(\tilde{u})=\hat{m}_{\tilde{V}}, \quad \partial_{\tilde{V}}(\tilde{u})=0 .
$$

Jointly with Lemma 2.7 , we have $I_{\tilde{V}}^{\prime}(\tilde{u})=0$. If $\tilde{V}>V_{0}$, then it follows from (6.27) that

$$
m_{0} \geq \lim _{n \rightarrow \infty} m_{\varepsilon_{n}}=\lim _{n \rightarrow \infty} I_{\varepsilon_{n}}\left(u_{\varepsilon_{n}}\right)=\hat{m}_{\tilde{V}}>m_{0} .
$$

This contradiction shows that

$$
\lim _{\varepsilon \rightarrow 0} V\left(\varepsilon y_{\varepsilon}\right)=V(\tilde{y})=V_{0}=\min _{x \in \mathbb{R}^{3}} V(x),
$$

and so $\tilde{u}$ is a ground state solution of $\left(K_{V_{0}}\right)$.

Proof of Theorem 1.2 Let $\bar{v}(x)=\bar{u}(x / \varepsilon)$ and $x_{\varepsilon}:=\varepsilon y_{\varepsilon}$. In the view of Lemma 5.4, for every $\varepsilon \in\left(0, \varepsilon_{0}\right], \bar{v}_{\varepsilon}$ is a ground state solution of $\left(S K_{\varepsilon}\right)$ which satisfies (1.6). Obviously, (i) follows from (6.40). Moreover, Lemma 6.7 and 6.8 imply that (ii) and (iii) hold.

Acknowledgements: The authors would like to thank anonymous referees for carefully reading this paper and making valuable comments and suggestions which help to improve the presentation of the paper greatly.

Supported by the SNSFC(ZR2020MA005).

Conflict of interest statement: Authors state no conflict of interest. 


\section{References}

[1] C. Alves, F. Corrêa and G. Figueiredo, On a class of nonlocal elliptic problems with critical growth, Differ. Equ. Appl. 2 (2010), no.3, 409-417.

[2] C. Alves, F. Corrêa and T. Ma, Positive solutions for a quasilinear elliptic equation of Kirchhoff type, Comput. Math. Appl. 49 (2005), no.1, 85-93.

[3] C. Alves and G. Figueiredo, Nonliear perturbations of a periodic Krichhoff equation in $\mathbb{R}^{N}$, Nonlinear Anal. 75 (2012), no.5, 2750-2759.

[4] A. Arosio and S. Panizzi, On the well-posedness of the Kirchhoff string, Trans. Amer. Math. Soc. 348 (1996), no.1, 305-330.

[5] T. Bartsch and Z. Wang, Existence and multiplicity results for superlinear elliptic problems on $\mathbb{R}^{N}$, Comm. Part Diff Eq. 20 (1995), no.9-10, 1725-1741.

[6] S. Chen, A. Fiscella, P. Pucci and X.H. Tang, Semiclassical ground state solutions for critical Schrödinger-Poisson systems with lower perturbations, J. Differ. Equ. 268 (2020), no.6, 2672-2716.

[7] S. Chen and X. Tang, Ground state sign-changing solutions for a class of Schrödinger-Poisson type problems in $\mathbb{R}^{3}, Z$. Angew. Math. Phys. 67 (2016), no.4, 1-18.

[8] S. Chen and X. Tang, On the planar Schrödinger-Poisson system with the axially symmetric potential, J. Differ. Equ. 268 (2020), no.3, 945-976.

[9] B. Cheng and X. Wu, Existence results of positive solutions of Kirchhoff type problems, Nonlinear Anal. 71 (2009), no.10, 4883-4892.

[10] Y. Deng, S. Peng and W. Shuai, Existence and asymptotic behavior of nodal solutions for the Kirchhoff-type problems in $\mathbb{R}^{3}$, J. Funct. Anal. 269 (2015), no.11, 3500-3527.

[11] Y. Ding and X. Liu, Semiclassical solutions of Schrödinger equations with magnetic fields and critical nonlinearities, Manuscripta Math. 140 (2013), no.1, 51-82.

[12] I. Ekeland, On the variational principle, J. Math. Anal. Appl. 47 (1974), no.2, 324-353.

[13] G. Figueiredo and J.R. Santos, Multiplicity and concentration behavior of positive solutions for a Schrödinger-Kirchhoff type problem via penalization method, ESAIM: Control. Optim. Calc. Var. 20 (2014), no.2, 389-415.

[14] Z. Guo, Ground states for Kirchhoff equations without compact condition, J. Differ. Equ. 259 (2015), no.7, 2884-2902.

[15] Y. He and G. Li, Standing waves for a class of Kirchhoff type problems in $\mathbb{R}^{3}$ involving critical Sobolev exponents, Calc. Var. Partial Differential Equations 54 (2005), no.3, 3067-3106.

[16] Y. He, G. Li and S. Peng, Concentrating bound states for Kirchhoff type problems in $\mathbb{R}^{3}$ involving critical sobolev exponents, Adv. Nonlinear Stud. 14 (2014), no.2, 483-510.

[17] X. He and W. Zou, Existence and concentration behavior of positive solutions for a Kirchhoff equation in $\mathbb{R}^{3}$. J. Differ. Equ. 252 (2012), no.2, 1813-1834.

[18] X. He and W. Zou, Ground states for nonlinear Kirchhoff equations with critical growth, Annali di Matematica Pura ed Applicata 193 (2014), no.2, 473-500.

[19] L. Jeanjean, On the existence of bounded Palais-Smale sequences and application to a Landsman-Lazer-type problem set on $\mathbb{R}^{N}$, Proc. Roy. Soc. Edingburgh Sect. A Math. 129 (1999), no.4, 787-809.

[20] G. Kirchhoff and K.Hensel, Vorlesungen über mathematische Physik, Vol.1, Teubner, Leipzig, 1883.

[21] G. Li and C. Wang, The existence of a nontrivial solution to a nonlinear elliptic problem of linking type without the Ambrosetti-Rabinowitz condition. Ann. Acad. Sci. Fenn. Mathematica. 36 (2011), no.2, 461-480.

[22] G. Li and H. Ye, Existence of positive ground state solutions for the nonlinear Kirchhoff type equations in $\mathbb{R}^{3}$, J. Differ. Equ. 57 (2014), no.2, 566-600.

[23] X. Lin and J. Wei, Existence and concentration of ground state solutions for a class of Kirchhoff-type problems. Nonlinear Anal. 195 (2020), 111715.

[24] J. Lions, On some questions in boundary value problems of mathmatical phisics, North-Holland Math. Stud. 30 (1978), 284-346.

[25] Z. Liu and S. Guo, Existence and concentration of positive ground states for a Kirchhoff equation involving critical Sobolev exponent, Z. Angew. Math. Phys. 66 (2015), no.3, 747-769.

[26] D. Lü and S. Peng, Existence and asymptotic behavior of vector solutions for coupled nonlinear Kirchhoff-type systems, J. Differ. Equ. 263 (2017), no.12, 8947-8978.

[27] T. Ma and J. Rivera, Positive solutions for a nonlinear nonlocal elliptic transmission problem, Appl. Math. Lett. 16 (2003), no.2, 243-248.

[28] A. Mao and Z. Zhang, Sign-changing and multiple solutions of Kirchhoff type problems without the P.S. condition, Nonlinear Anal. 70 (2009), no.3, 1275-1287.

[29] A. Pankov, On decay of solutions to nonlinear Schrödinger equations, Proc. Amer. Math. Soc. 136 (2008), no.7, $2565-2570$.

[30] K. Perera and Z. Zhang, Nontrivial solutions of Kirchhoff-type problems via the Yang index, J. Differ. Equ. 221 (2006), no.1, 246-255.

[31] D. Ruiz, The Schrödinger-Poisson equation under the effect of a nonlinear local term, J. Funct. Anal. 237 (2006), no.2, 655674. 
[32] W. Shuai, Sign-changing solutions for a class of Kirchhoff-type problem in bounded domains, J. Differ. Equ. 259 (2015), no.2, 1256-1274.

[33] B. Simon, Schrödinger semigroups, Bull. Amer. Math. Soc. 7 (1982), no.2, 447-526.

[34] J. Sun and C. Tang, Resonance problems for Kirchhoff type equations, Discrete Contin. Dyn. Syst. 33 (2013), no.5, 2139 2154.

[35] X. Tang and S. Chen, Singularly perturbed choquard equations with nonlinearity satisfying Berestycki-Lions assumptions, Adv. Nonlinear Anal. 9 (2020), no.1, 413-437.

[36] X. Tang and S. Chen, Ground state solutions of Nehari-Pohožaev type for Kirchhoff-type problems with general potentials, Calc. Var. Partial Differential Equations, 56 (2017), no.4, 110-134.

[37] J. Wang, L. Tian, J. Xu and F. Zhang, Multiplicity and concentration of positive solutions for a Kirchhoff type problem with critical growth, J. Differ. Equ. 253 (2012), no.7, 2314-2351.

[38] M. Willem, Minimax theorems, Progress in Nonlinear Differential Equations and their Applications, Vol. 24, Birkhäuser Boston Inc. Boston, 1996.

[39] J. Zhang, Z. Chen and W. Zou, Standing waves for nonlinear Schrödinger equations involving critical growth, J. Lond. Math. Soc. 90 (2014), no.9, 827-844. 